\title{
Loss-tolerant concatenated Bell-state measurement with encoded coherent-state qubits for long-range quantum communication
}

\author{
Seok-Hyung Lee $\odot,{ }^{1}$ Seung-Woo Lee, ${ }^{2}$ and Hyunseok Jeong ${ }^{1, *}$ \\ ${ }^{1}$ Department of Physics and Astronomy, Seoul National University, Seoul 08826, Republic of Korea \\ ${ }^{2}$ Center for Quantum Information, Korean Institute of Science and Technology, Seoul 02792, Republic of Korea
}

(Received 22 February 2021; revised 27 July 2021; accepted 19 November 2021; published 21 December 2021)

\begin{abstract}
The coherent-state qubit is a promising candidate for optical quantum information processing due to its nearly deterministic nature of the Bell-state measurement (BSM). However, its nonorthogonality incurs difficulties such as the failure of the BSM. One may use a large amplitude $(\alpha)$ for the coherent state to minimize the failure probability, but the qubit then becomes more vulnerable to dephasing by photon loss. We propose a hardware-efficient concatenated BSM (CBSM) scheme with modified parity encoding using coherent states with reasonably small amplitudes $(|\alpha| \lesssim 2)$, which simultaneously suppresses both failures and dephasing in the BSM procedure. We numerically show that the CBSM scheme achieves a success probability arbitrarily close to unity for appropriate values of $\alpha$ and sufficiently low photon loss rates (e.g., $\lesssim 5 \%$ ). Furthermore, we verify that the quantum repeater scheme exploiting the CBSM scheme for quantum error correction enables one to carry out efficient long-range quantum communication over $1000 \mathrm{~km}$. We show that the performance is comparable to those of other up-to-date methods and even exceeds them in some cases. Finally, we present methods to prepare logical qubits under modified parity encoding, and we implement elementary logical operations, which consist of several physical-level ingredients such as generation of superpositions of coherent states (SCSs) and elementary gates under the coherent-state basis. We then estimate the effects of imperfect physical-level elements on the performance of the scheme. Our work demonstrates that the encoded coherent-state qubits in free-propagating fields provide an alternative route to fault-tolerant information processing, especially to long-range quantum communication.
\end{abstract}

DOI: 10.1103/PhysRevResearch.3.043205

\section{INTRODUCTION}

Optical systems are a competitive candidate for quantum information processing (QIP) due to their long coherence time and advantages in long-distance transmission [1]. It is well known that they are particularly promising for quantum communication. Single-photon states are usually considered for the carriers of optical qubits such as vacuum-single-photonqubits (single-rail encoding) [2] and polarization qubits (dual-rail encoding) [3]. However, these encoding schemes have a drawback in that the Bell-state measurement (BSM) is nondeterministic with linear optics [4,5]. The BSM is essential for QIP tasks such as quantum teleportation [6,7] and entanglement swapping [8,9]. Quantum teleportation is widely employed not only for quantum communication but also for all-optical quantum computation with gate teleportation [3]. It is thus important to overcome the problem of nondeterministic BSM. A number of methods have been suggested using multiple photons for encoding [10,11], ancillary

\footnotetext{
*h.jeong37@gmail.com

Published by the American Physical Society under the terms of the Creative Commons Attribution 4.0 International license. Further distribution of this work must maintain attribution to the author(s) and the published article's title, journal citation, and DOI.
}

states [12-14], coherent states [15-24], and hybrid states [25-27] to improve the success probability of BSM. Among them, we focus here on the scheme using coherent-state qubits that enables one to perform a nearly deterministic BSM with linear optics [15,16,28].

Early studies on coherent states as carriers of quantum information focus on how to construct logical qubits and elementary logical gates $[15,17,18,29-32]$. In these works, the basis set is chosen either as $\{| \pm \alpha\rangle\}$ or as $\left\{N_{ \pm}(|\alpha\rangle \pm|-\alpha\rangle)\right\}$, where $| \pm \alpha\rangle$ are coherent states of amplitudes $\pm \alpha$, and $N_{ \pm}$are normalization factors. Various attempts to obtain fault-tolerance on QIP with coherent states have been made, starting from simple embedding on well-known discretevariable (DV) encoding schemes [19,22], to exploiting the property of continuous-variable (CV) systems [24,33-38], with some experimental demonstration [39-42]. Recently, it was claimed that a simple one-dimensional (1D) repetition cat code enables hardware-efficient topologically protected quantum computation by exploiting the 2D phase space for logical operations [43]. However, these studies mainly deal with coherent-state qubits inside a cavity system, and they cannot be directly applied to fault-tolerant QIP in free-propagating optical fields. Our main goal is to investigate the possibility of using simple concatenated repetition codes, which can be generated and manipulated with combinations of well-known elementary gates, for fault-tolerant QIP with free-propagating coherent-state qubits. 
As mentioned before, the BSM of coherent-state qubits, where the basis is $\{| \pm \alpha\rangle\}$, is nearly deterministic. However, due to the nonorthogonality of the basis set, a small but non-negligible probability of failure exists $[15,16]$. One may use coherent states with large values of $|\alpha|$ to solve this problem, but the qubit then becomes more vulnerable to dephasing by photon loss [19]. It is impossible to ideally suppress both failures and dephasing simultaneously with such an elementary coherent-state encoding. In this paper, motivated by recent works on the concatenated Bell-state measurement (CBSM) with multiphoton polarization qubits [11,44] and the repetition cat code [43], we overcome these obstacles by introducing the CBSM with the modified parity encoding employing coherent states. We propose an elaborately designed CBSM scheme with consideration of hardware efficiency, and we show numerically that the scheme successfully suppresses both failures and dephasing simultaneously with reasonably small amplitudes (e.g., $|\alpha|<2$ ) of coherent states.

One of the key applications with BSMs is long-distance quantum communication through quantum repeaters [45]. In the initially proposed quantum repeater schemes to generate Bell pairs between distant parties [46-50], heralded entanglement generation is required for suppressing transmission errors, which makes long-lived quantum memory essential [51]. Recently, quantum repeater schemes exploiting quantum error correction (QEC) have been suggested for suppressing errors, which do not require long-lived quantum memory $[11,44,51-58]$. In these schemes, a quantum repeater is built up by encoding information with QEC codes, sending it by a lossy channel, and relaying the encoded information from each station to the next station with error correction. In each repeater station, a fault-tolerant BSM may be used for QEC; each incoming lossy logical qubit is teleported out of the station while recovering its original information [11]. Later in this paper, we evaluate the performance of the quantum repeater scheme using our CBSM scheme, and we show that it indeed enables quantum repeaters with high performance over distances longer than $1000 \mathrm{~km}$.

The outline of the paper is as follows. In Sec. II, we review the BSM scheme of lossless coherent-state qubits, generalize it to lossy cases, and evaluate its success, failure, and error rates against the coherent-state amplitude $\alpha$ and the photon survival rate. In Sec. III, we present the modified parityencoding scheme employing coherent-state qubits, and we show the hierarchy relation between the logical, block, and physical levels. In Sec. IV, we first suggest an unoptimized CBSM scheme, which uses simple majority votes and counting of measurement results only, and we analyze the root of its fault tolerance. After that, we propose an improved CBSM scheme that is elaborately designed considering hardware efficiency. In Sec. V, we present the analytic expressions of the probability distributions of CBSM results, which are simple matrix forms enabling fast sampling of the results and can be generalized to any CBSM scheme. In Sec. VI, we show the results of numerical calculations. We first analyze the success, failure, and error probabilities of a CBSM. We then investigate the performance of the quantum repeater scheme, which uses our CBSM scheme for error correction, as one of the key applications of the BSM. In Sec. VII, we describe the methods to implement our encoding and CBSM schemes in terms of

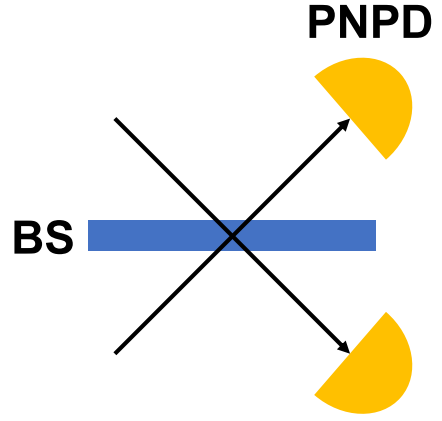

FIG. 1. Bell-state measurement (BSM) scheme of coherent-state qubits [15] with one 50:50 beam splitter (BS) and two photonnumber parity detectors (PNPDs). The result is determined by the measurement results of the PNPDs as shown in Eq. (2).

physical-level elements such as superpositions of coherent states (SCSs) and logical gates on coherent-state qubits. We then briefly review recent progress on implementing these elements and roughly estimate the effects of experimental imperfections. We conclude with final remarks in Sec. VIII.

\section{BELL-STATE MEASUREMENT OF LOSSY COHERENT-STATE QUBITS}

We first review the BSM scheme of lossless coherent-state qubits encoded with the basis

$$
\left|0_{L}\right\rangle:=|\alpha\rangle, \quad\left|1_{L}\right\rangle:=|-\alpha\rangle .
$$

The four Bell states of coherent-state qubits are

$$
\begin{aligned}
& \left|\phi_{ \pm}\right\rangle:=N_{ \pm}(|\alpha\rangle|\alpha\rangle \pm|-\alpha\rangle|-\alpha\rangle), \\
& \left|\psi_{ \pm}\right\rangle:=N_{ \pm}(|\alpha\rangle|-\alpha\rangle \pm|-\alpha\rangle|\alpha\rangle),
\end{aligned}
$$

where $N_{ \pm}:=\left[2\left(1 \pm e^{-4|\alpha|^{2}}\right)\right]^{-1 / 2}$ are normalization factors. A BSM of lossless coherent-state qubits is performed with a 50:50 beam splitter and two photon number parity detectors (PNPDs) [15,16], as shown in Fig. 1. The four Bell states can be deterministically identified from the results of the PNPDs unless both of the PNPDs do not detect any photons:

$$
\begin{array}{rlrl}
(\text { even, } 0) & \rightarrow\left|\phi_{+}\right\rangle, & (\text {odd }, 0) & \rightarrow\left|\phi_{-}\right\rangle, \\
(0, \text { even }) & \rightarrow\left|\psi_{+}\right\rangle, & (0, \text { odd }) \rightarrow\left|\psi_{-}\right\rangle .
\end{array}
$$

In the case in which both of the PNPDs do not detect photons (regarded as the failure of the BSM), only the sign of the Bell state (namely, " \pm " of $\left|\phi_{ \pm}\right\rangle$or $\left|\psi_{ \pm}\right\rangle$) can be determined since there exists ambiguity between $\left|\phi_{+}\right\rangle$and $\left|\psi_{+}\right\rangle$.

For realistic scenarios, we need to introduce photon loss. We use the photon loss model by the Master equation under the Born-Markov approximation with zero temperature [59]:

$$
\frac{\partial \rho}{\partial \tau}=\gamma \sum_{i}\left(\hat{a}_{i} \rho \hat{a}_{i}^{\dagger}-\frac{1}{2} \hat{a}_{i}^{\dagger} \hat{a}_{i} \rho-\frac{1}{2} \rho \hat{a}_{i}^{\dagger} \hat{a}_{i}\right),
$$

where $\rho(\tau)$ is the density operator of the system suffering photon loss as a function of time $\tau, \gamma$ is the decay constant, and $\hat{a}_{i}$ is the annihilation operator of the $i$ th mode. It is known that this photon loss model is equivalent to the beam splitter model where each mode is independently mixed with the vacuum state by a beam splitter with the transmittance $t=e^{-\gamma \tau / 2}$ 
and the reflectance $r=\sqrt{1-t^{2}}$ [60]:

$$
\left(\begin{array}{l}
\hat{a} \\
\hat{b}
\end{array}\right) \rightarrow\left(\begin{array}{l}
\hat{a}^{\prime} \\
\hat{b}^{\prime}
\end{array}\right)=\left(\begin{array}{cc}
t & -r \\
r & t
\end{array}\right)\left(\begin{array}{l}
\hat{a} \\
\hat{b}
\end{array}\right) .
$$

Here, $\hat{a}\left(\hat{a}^{\prime}\right)$ is the annihilation operator of the input (output) mode of each concerned system, and $\hat{b}\left(\hat{b}^{\prime}\right)$ is that of the input (output) mode of an ancillary system which is initially in the vacuum state. The final state after suffering photon loss is obtained by tracing out the ancillary system from the output state of the beam splitter. Since the photon survival rate $\eta$ is equal to $t^{2}$, the final state can be expressed in terms of $\eta$.

Now, we consider the BSM on lossy coherent-state qubits. More precisely, we deal with a situation in which the two coherent-state qubits suffer photon loss before the BSM in Fig. 1 is performed. We first rewrite each element of the BSM scheme mathematically: $\mathcal{U}_{\mathrm{BS}}$ is a unitary channel corresponding to a 50:50 beam splitter, $\Lambda_{\eta}$ is a photon loss channel with a survival rate $\eta$, and $\Pi_{x}$ for $x \in\{0,1,2\}$ is a projector defined by

$$
\begin{aligned}
\Pi_{0} & :=\left|0_{\mathrm{F}}\right\rangle\left\langle 0_{\mathrm{F}}\left|, \quad \Pi_{1}:=\sum_{n \geqslant 1: \text { odd }}\right| n_{\mathrm{F}}\right\rangle\left\langle n_{\mathrm{F}}\right|, \\
\Pi_{2} & :=\sum_{n \geqslant 2: \text { even }}\left|n_{\mathrm{F}}\right\rangle\left\langle n_{\mathrm{F}}\right|,
\end{aligned}
$$

where $\left|n_{\mathrm{F}}\right\rangle$ is the Fock state with a photon number of $n$. Then a set of operators

$$
M_{x, y}:=\left[\mathcal{U}_{\mathrm{BS}} \circ\left(\Lambda_{\eta_{1}} \otimes \Lambda_{\eta_{2}}\right)\right]^{\dagger}\left(\Pi_{x} \otimes \Pi_{y}\right)
$$

with $x, y \in\{0,1,2\}$ forms a positive-operator valued measure (POVM) corresponding to the BSM of the lossy coherent-state qubits. Their explicit forms are presented in Appendix A.

Assuming the equal prior probability distribution of the four Bell states $\mathcal{B}_{0}=\left\{\left|\phi_{ \pm}\right\rangle,\left|\psi_{ \pm}\right\rangle\right\}$, we choose the Bell state $|B\rangle \in \mathcal{B}_{0}$ maximizing the posterior probability from the PNPD results given by $(x, y)$ :

$$
\begin{aligned}
\operatorname{Pr}(B \mid x, y) & =\frac{\operatorname{Pr}(x, y \mid B) \operatorname{Pr}(B)}{\sum_{\left|B^{\prime}\right\rangle \in \mathcal{B}_{0}} \operatorname{Pr}\left(x, y \mid B^{\prime}\right) \operatorname{Pr}\left(B^{\prime}\right)} \\
& \propto \operatorname{Pr}(x, y \mid B)=\left\langle B\left|M_{x, y}\right| B\right\rangle .
\end{aligned}
$$

In other words, we choose $|B\rangle \in \mathcal{B}_{0}$ satisfying

$$
|B\rangle=\underset{\left|B^{\prime}\right\rangle \in \mathcal{B}_{0}}{\operatorname{argmax}}\left\langle B^{\prime}\left|M_{x, y}\right| B^{\prime}\right\rangle,
$$

for the result of the BSM. Table I shows the correspondences between the pairs of the PNPD results and the resulting Bell states, which are obtained from Eq. (6) and the POVM elements of the BSM in Appendix A. Note that some cases that never happen in lossless cases occur in lossy cases: Both $x$ and $y$ can be nonzero at the same time, while the probabilities of these cases vanish for $\eta_{1}=\eta_{2}$.

If the state before suffering the photon loss is one of the four Bell states, there are five possible cases regarding the result of the measurement: success, $X$-error, $Z$-error, $Y$-error, and failure. If the Bell state obtained from Eq. (6) is the same as the initial one, we call it a success. An $X$-error corresponds to a "letter flip," i.e., the change of the letter (" $\phi$ " or " $\psi$ ") of a Bell state such as from $\left|\phi_{+}\right\rangle$to $\left|\psi_{+}\right\rangle$. A $Z$-error corresponds to a "sign flip," i.e., the change of the sign (" \pm ") of a Bell
TABLE I. Correspondences between the pairs of the PNPD results and the resulting Bell states. The Bell state $|B\rangle \in\left\{\left|\phi_{ \pm}\right\rangle,\left|\psi_{ \pm}\right\rangle\right\}$ is chosen to maximize the posterior probability $\operatorname{Pr}(B \mid x, y)$ in Eq. (5). Here, $x$ and $y$ indicate the results of the two PNPDs, where 0,1 , and 2 denote zero, odd, and even detection, respectively. The cases when both $x$ and $y$ are nonzero can occur only when the loss rates of the two modes are different. We also note that only the sign of the Bell state can be determined in the cases of $x=y$, which we call a "failure," since both $\left|\phi_{+}\right\rangle$and $\left|\psi_{+}\right\rangle$maximize the posterior probability at the same time.

\begin{tabular}{ccccc}
\hline \hline & $\mathrm{y}$ & 0 & 1 & \\
\hline 0 & & & & \\
1 & & $\phi_{+}$or $\psi_{+}$ & $\psi_{-}$ & $\psi_{+}$ \\
2 & $\phi_{-}$ & $\phi_{+}$or $\psi_{+}$ & $\psi_{-}$ \\
\hline \hline & $\phi_{+}$ & $\phi_{-}$ & $\phi_{+}$or $\psi_{+}$ \\
\hline
\end{tabular}

state such as from $\left|\phi_{+}\right\rangle$to $\left|\phi_{-}\right\rangle$. A $Y$-error corresponds to simultaneous symbol and sign flips. The last case, a failure, corresponds to the cases of $x=y$ in Table I where the letter of the Bell state cannot be determined, since both $\left|\phi_{+}\right\rangle$and $\left|\psi_{+}\right\rangle$maximize the posterior probability at the same time. We would like to emphasize that the sign can still be determined even if a BSM fails.

Now, we numerically analyze the success, failure, and error probabilities of a BSM on coherent-state qubits. We assume that both systems suffer internal losses with the survival rate of $\eta_{0}$, and the photons of the second system travel the distance of $L_{0}=1 \mathrm{~km}$ before the measurement. The photon survival rates of the two systems are then $\eta_{1}:=\eta_{0}$ and $\eta_{2}:=$ $\eta_{0} e^{-L_{0} / L_{\text {att }}}$, respectively, where $L_{\text {att }}=22 \mathrm{~km}$ is the attenuation length.

Figure 2 shows the success, failure, and error probabilities of the BSM in this situation against the amplitude $\alpha$ of the coherent state and the internal photon survival rate $\eta_{0}$. It shows the well-known fact that the success probability is higher than that of a BSM on multiphoton polarization qubits with the same photon number. Also, the failure and $Z$-error probabilities have a tradeoff relation with changing $\alpha$ : When $\alpha$ increases, failures get less probable while $Z$-errors get more probable. It is because coherent states with large amplitudes have smaller overlaps with the vacuum state and are more vulnerable to dephasing by photon loss. Furthermore, it is worth noting that the errors are strongly biased, i.e., the $X$ and $Y$-error probabilities are much smaller than the failure and $Z$-error probabilities regardless of the values of $\alpha$ and $\eta_{0}$ : $p_{X}, p_{Y} \lesssim 10^{-4}$. They even vanish if $\eta_{1}=\eta_{2}$ since both $x$ and $y$ in Table I can be nonzero simultaneously only when the two photon survival rates are different, which is particularly important for constructing a hardware-efficient CBSM scheme in Sec. IV C.

\section{MODIFIED PARITY ENCODING SCHEME WITH COHERENT-STATE QUBITS}

Now, we present the encoding scheme used in our CBSM scheme. We modify the parity state encoding or generalized Shor's encoding [11,61] for the coherent-state qubit. The modified parity encoding is defined as follows: 


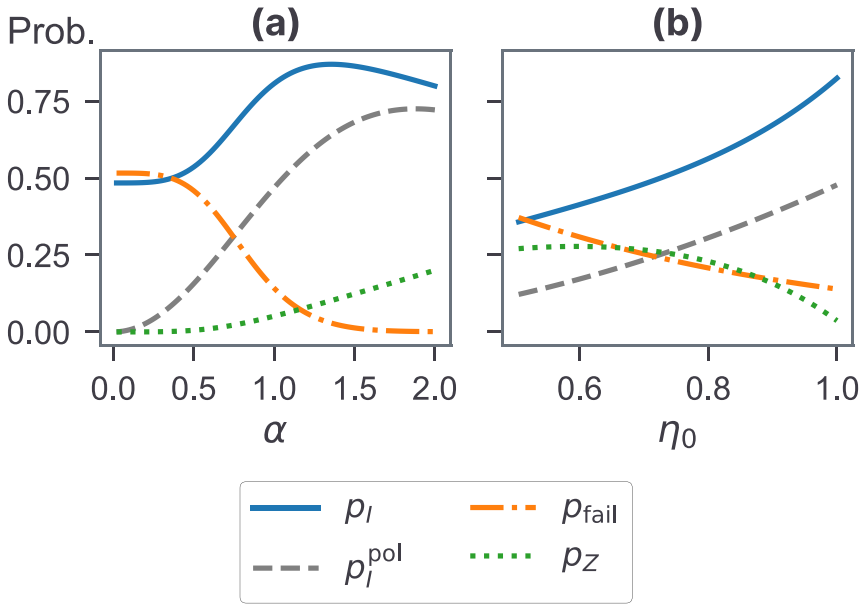

FIG. 2. Success, failure, and Z-error probabilities $\left(p_{i}, p_{\text {fail }}\right.$, and $p_{Z}$, respectively) of a BSM on coherent-state qubits against (a) $\alpha$ (fixing $\eta_{0}=0.99$ ) or (b) $\eta_{0}$ (fixing $\alpha=1$ ), where $\alpha$ is the amplitude of the used coherent states and $\eta_{1}:=\eta_{0}$ is the photon survival rate of the first system. The photon survival rate of the second system is set to be $\eta_{2}:=\eta_{0} e^{-L / L_{\text {att }}}$, where $L:=1 \mathrm{~km}$ and $L_{\text {att }}:=22 \mathrm{~km}$. It corresponds to the situation when both systems suffer internal losses with the photon survival rates of $\eta_{0}$, and the photons of the second system travel the distance of $L:=1 \mathrm{~km}$ before the measurement. We also present the success probability $p_{I}^{\text {pol }}$ of a BSM on multiphoton polarization qubits for different photon numbers [10], which is plotted for comparison, where $\alpha$ is now the amplitude of the coherent state which has the same photon number with each qubit. The $X$ and $Y$-error probabilities ( $p_{X}$ and $p_{Y}$, respectively) are not plotted, since they are much smaller than other probabilities regardless of $\alpha$ and $\eta_{0}: p_{X}, p_{Y} \lesssim 10^{-4}$.

Definition 1. The logical basis $\left\{\left|0_{L}\right\rangle,\left|1_{L}\right\rangle\right\}$ of the $(n, m, \alpha)$ modified parity encoding where $n$ and $m$ are odd integers and $\alpha$ is a complex number is defined as

$$
\begin{aligned}
& \left|0_{L}\right\rangle:=\left[N^{(m)}\left\{|\tilde{+}\rangle^{\otimes m}+|\tilde{\sim}\rangle^{\otimes m}\right\}\right]^{\otimes n}, \\
& \left|1_{L}\right\rangle:=\left[N^{(m)}\left\{|\tilde{+}\rangle^{\otimes m}-|\tilde{\sim}\rangle^{\otimes m}\right\}\right]^{\otimes n},
\end{aligned}
$$

where $|\widetilde{ \pm}\rangle:=|\alpha\rangle \pm|-\alpha\rangle$ are unnormalized SCSs (the tilde above the ket is used to denote that it is unnormalized) and $N^{(m)}:=\left[2^{m}\left\{\left(1+e^{-2|\alpha|^{2}}\right)^{m}+\left(1-e^{-2|\alpha|^{2}}\right)^{m}\right\}\right]^{-1 / 2}$.

The SCS $|\tilde{+}\rangle(|\tilde{-}\rangle)$ is called an even (odd) SCS because it contains an even (odd) number of photons. Note that the encoding coincides the original coherent-state encoding in Eq. (1) if $n=m=1$.

The overlap between $\left|0_{L}\right\rangle$ and $\left|1_{L}\right\rangle$ is given as

$$
\begin{aligned}
\left\langle 0_{L} \mid 1_{L}\right\rangle & =\left(\frac{\left(1+e^{-2|\alpha|^{2}}\right)^{m}-\left(1-e^{-2|\alpha|^{2}}\right)^{m}}{\left(1+e^{-2|\alpha|^{2}}\right)^{m}+\left(1-e^{-2|\alpha|^{2}}\right)^{m}}\right)^{n} \\
& \approx\left(m e^{-2|\alpha|^{2}}\right)^{n},
\end{aligned}
$$

where the approximation holds when $m e^{-2|\alpha|^{2}} \ll 1$. If $\alpha>1$, $n$ does not need to be very large to make the basis nearly orthogonal. For example, fixing $m$ to be $5,\left\langle 0_{L} \mid 1_{L}\right\rangle \lesssim 0.001$ for $\alpha=1.2$ and $n \geqslant 7$, and $\left\langle 0_{L} \mid 1_{L}\right\rangle \lesssim 10^{-5}$ for $\alpha=1.6$ and $n \geqslant 3$.

The modified parity encoding has a hierarchy structure of Hilbert spaces: the logical, block, and physical levels.
The logical-level space is the total Hilbert space spanned by $\left\{\left|0_{L}\right\rangle,\left|1_{L}\right\rangle\right\}$. It can be divided into $n$ block-level spaces (referred to as blocks), each of which is spanned by $\left\{\left| \pm^{(m)}\right\rangle\right\}$, where $\left| \pm^{(m)}\right\rangle:=N^{(m)}\left\{|\tilde{+}\rangle^{\otimes m} \pm|\tilde{-}\rangle^{\otimes m}\right\}$. A block is again divided into $m$ physical-level spaces (referred to as $P L S s$ ), each of which is spanned by $\{| \pm \alpha\rangle\}$.

We also define the four Bell states for each level as follows, omitting normalization constants:

(a) Logical level:

$$
\begin{aligned}
& \left|\Phi_{ \pm}\right\rangle:=\left|0_{L}\right\rangle\left|0_{L}\right\rangle \pm\left|1_{L}\right\rangle\left|1_{L}\right\rangle, \\
& \left|\Psi_{ \pm}\right\rangle:=\left|0_{L}\right\rangle\left|1_{L}\right\rangle \pm\left|1_{L}\right\rangle\left|0_{L}\right\rangle .
\end{aligned}
$$

(b) Block level:

$$
\begin{aligned}
\left|\phi_{ \pm}^{(m)}\right\rangle & :=\left|+{ }^{(m)}\right\rangle\left|+^{(m)}\right\rangle \pm\left|-^{(m)}\right\rangle\left|-{ }^{(m)}\right\rangle, \\
\left|\psi_{ \pm}^{(m)}\right\rangle & :=\left|+{ }^{(m)}\right\rangle\left|-{ }^{(m)}\right\rangle \pm\left|-^{(m)}\right\rangle\left|+{ }^{(m)}\right\rangle .
\end{aligned}
$$

(c) Physical level:

$$
\begin{aligned}
\left|\phi_{ \pm}\right\rangle & :=|\alpha\rangle|\alpha\rangle \pm|-\alpha\rangle|-\alpha\rangle, \\
\left|\psi_{ \pm}\right\rangle & :=|\alpha\rangle|-\alpha\rangle \pm|-\alpha\rangle|\alpha\rangle .
\end{aligned}
$$

Each logical-level Bell state can be decomposed into blocklevel Bell states:

$$
\begin{aligned}
& \left|\Phi_{+(-)}\right\rangle=\tilde{N}_{ \pm, n, m} \sum_{k=\text { even(odd) } \leqslant n} \mathcal{P}\left[\left|\widetilde{\phi_{-}^{(m)}}\right\rangle^{\otimes k}\left|\widetilde{\phi_{+}^{(m)}}\right\rangle^{\otimes n-k}\right], \\
& \left|\Psi_{+(-)}\right\rangle=\tilde{N}_{ \pm, n, m} \sum_{k=\text { even(odd }) \leqslant n} \mathcal{P}\left[\left|\widetilde{\psi_{-}^{(m)}}\right\rangle^{\otimes k}\left|\widetilde{\psi_{+}^{(m)}}\right\rangle^{\otimes n-k}\right],
\end{aligned}
$$

where

$$
\begin{gathered}
\tilde{N}_{ \pm, n, m}:=\frac{1}{\sqrt{2^{n-1}}}\left[1 \pm u(\alpha, m)^{2 n}\right]^{-\frac{1}{2}}, \\
\left.\widetilde{\phi_{ \pm}^{(m)}}\right\rangle:=\left[1 \pm u(\alpha, m)^{2}\right]^{\frac{1}{2}}\left|\phi_{ \pm}^{(m)}\right\rangle, \\
\widetilde{\left.\psi_{ \pm}^{(m)}\right\rangle}:=\left[1 \pm u(\alpha, m)^{2}\right]^{\frac{1}{2}}\left|\psi_{ \pm}^{(m)}\right\rangle, \\
u(\alpha, m):=\frac{\left(1+e^{-2|\alpha|^{2}}\right)^{m}-\left(1-e^{-2|\alpha|^{2}}\right)^{m}}{\left(1+e^{-2|\alpha|^{2}}\right)^{m}+\left(1-e^{-2|\alpha|^{2}}\right)^{m}},
\end{gathered}
$$

and $\mathcal{P}[\cdot]$ is the summation of all the possible permutations of the tensor product inside the square brackets.

Similarly, each block-level Bell state can be decomposed into physical-level Bell states:

$$
\begin{aligned}
\left|\phi_{ \pm}^{(m)}\right\rangle & =\frac{\tilde{N}_{ \pm, 1, m}}{\sqrt{2}} \sum_{l=\text { even } \leqslant m} \mathcal{P}\left[\left|\psi_{ \pm}\right\rangle^{\otimes l}\left|\phi_{ \pm}\right\rangle^{\otimes m-l}\right], \\
\left|\psi_{ \pm}^{(m)}\right\rangle & =\frac{\tilde{N}_{ \pm, 1, m}}{\sqrt{2}} \sum_{l=\text { odd } \leqslant m} \mathcal{P}\left[\left|\psi_{ \pm}\right\rangle^{\otimes l}\left|\phi_{ \pm}\right\rangle^{\otimes m-l}\right] .
\end{aligned}
$$

The core of the CBSM is contained in Eqs. (8) and (12); they make it possible to perform a logical BSM by the combination of $n$ block-level BSMs, each of which is again performed by the combination of $m$ physical-level BSMs.

The equations also show that, in a lossless system, a CBSM does not incur any logical error (i.e., the only possible cases are success and failure). This property is important since failures are detectable whereas logical errors are not. Hence, the modified parity encoding in Definition 1 is the natural 


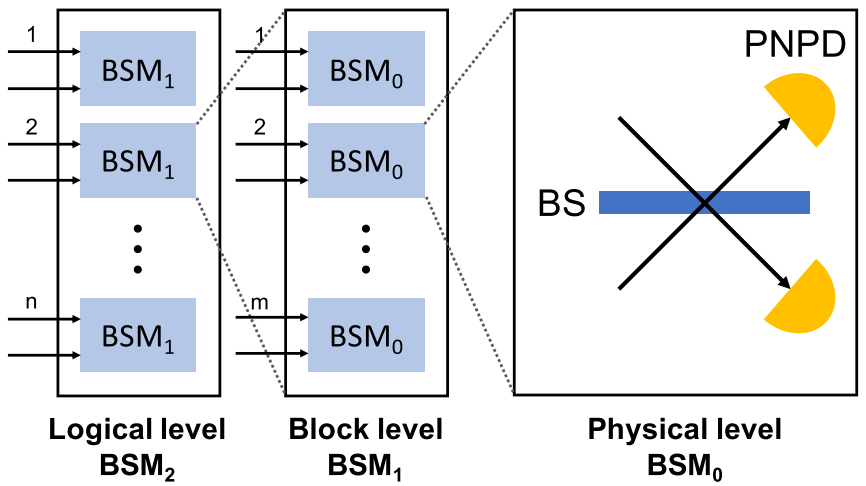

FIG. 3. Schematic figure of the CBSM schemes with coherentstate qubits. The schemes are done in a concatenated manner: Each logical-level BSM $\left(\mathrm{BSM}_{2}\right)$ is done by the combination of $n$ blocklevel BSMs $\left(\mathrm{BSM}_{1}\right.$ 's). Each $\mathrm{BSM}_{1}$ is again done by the combination of $m$ physical-level BSMs $\left(\mathrm{BSM}_{0}\right.$ 's).

extension of the original coherent-state encoding in Eq. (1), in the sense that this desired property still remains. If we use other states such as normalized SCSs or coherent states in place of unnormalized SCSs $|\widetilde{ \pm}\rangle$ for the encoding, this property no longer exists.

\section{CONCATENATED BELL-STATE MEASUREMENT WITH ENCODED COHERENT-STATE QUBITS}

Now, we suggest the concatenated Bell-state measurement (CBSM) schemes with the modified parity encoding presented in the previous section. The schematic figure of the CBSM schemes is shown in Fig. 3. As mentioned in the previous section, each logical-level BSM is done by the composition of $n$ block-level BSMs, and each block-level BSM is done by the composition of $m$ physical-level BSMs. We first consider an unoptimized scheme consisting of the simple counting of measurement results. We then present a hardware-efficient scheme that can significantly reduce the expected cost of the CBSM defined in terms of the expected number of physicallevel BSMs used for a single CBSM.

\section{A. Unoptimized CBSM scheme}

Here, we suggest a CBSM scheme that is unoptimized but much simpler than the hardware-efficient scheme presented in the next subsection. It is straightforward to justify the scheme with Eqs. (8) and (12). The interpretation of the measurement results in the scheme is summarized in Table II.

\section{Physical level: $\mathbf{B S M}_{0}$}

For a physical-level BSM (referred to as a $\mathrm{BSM}_{0}$ ), we use the BSM scheme for a single lossy coherent-state qubit presented in Fig. 1 and Table I. Remark that the sign of the Bell state is always determinable, while its letter is not determinable if the results of the two PNPDs are the same (namely, $x=y$ in Table I).

\section{Block level: $\mathrm{BSM}_{1}$}

A block-level BSM (referred to a $\mathrm{BSM}_{1}$ ) is done by performing a $\mathrm{BSM}_{0}$ on each PLS in the block. The sign of the block-level Bell state is determined by the majority vote of the signs of the $\mathrm{BSM}_{0}$ results. Its letter is determined by the parity of the number of the $\mathrm{BSM}_{0}$ results with the $\psi$ symbol: $\phi(\psi)$ if the number is even (odd).

Since $m$ is odd, the sign of the block-level Bell state is always determinable. Its letter is not determined if at least one $\mathrm{BSM}_{0}$ fails, which we regard as the $\mathrm{BSM}_{1}$ failing.

\section{Logical level: $\mathbf{B S M}_{2}$}

A logical-level BSM (referred to as a $\mathrm{BSM}_{2}$ ) is done by performing a $\mathrm{BSM}_{1}$ on each block. The sign of the logicallevel Bell state is determined by the parity of the number of the $\mathrm{BSM}_{1}$ results with the minus sign: plus (minus) if the number is even (odd). Its letter is determined by the majority vote of the letters of the $\mathrm{BSM}_{1}$ results excluding the failed ones.

Again, the sign of the logical-level Bell state is always determinable. Its letter is not determined if all the $\mathrm{BSM}_{1}$ 's fail or the resulting block-level Bell states have the same number of both letters. We regard such a case as the failure of the $\mathrm{BSM}_{2}$.

\section{B. Fault tolerance of concatenated Bell-state measurement}

We next investigate the fault tolerance of the unoptimized CBSM scheme suggested above. We argue that the physicaland block-level repetitions contribute to suppressing logical errors and failures, respectively.

First, a $Z(X)$-error in the logical level is suppressed by the majority vote in the block (logical) level. Note that the sign (letter) of a logical-level Bell state is determined only by the signs (letters) of the Bell states of the lower levels, as described in Table II. Z-errors (sign flips) in the

TABLE II. Interpretation of the measurement results in the unoptimized CBSM scheme. It is also valid in the hardware-efficient CBSM scheme, if we consider the results of $\mathrm{BSM}_{0}$ 's $\left(\mathrm{BSM}_{1}\right.$ 's) and $\mathrm{BSM}_{0}^{\text {sign }}$ 's $\left(\mathrm{BSM}_{1}^{\text {sign }}\right.$ 's) together when determining the sign of each block (logical) level Bell state.

\begin{tabular}{|c|c|c|}
\hline Level & $\operatorname{Sign}( \pm)$ & Letter $(\phi$ or $\psi)$ \\
\hline Physical $\left(\mathrm{BSM}_{0}\right)$ & \multicolumn{2}{|c|}{ BSM scheme of the original coherent-state qubits } \\
\hline Block $\left(\mathrm{BSM}_{1}\right)$ & Majority vote of the signs of the $\mathrm{BSM}_{0}$ results & $\begin{array}{l}\text { Number of } \mathrm{BSM}_{0} \text { results with the (-) sign: } \\
\phi \text { if even, } \psi \text { if odd }\end{array}$ \\
\hline Logical $\left(\mathrm{BSM}_{2}\right)$ & $\begin{array}{l}\text { Number of } \mathrm{BSM}_{1} \text { results with the } \psi \text { letter: } \\
(+) \text { if even, }(-) \text { if odd }\end{array}$ & Majority vote of the letters of the $\mathrm{BSM}_{1}$ results \\
\hline
\end{tabular}


(a)

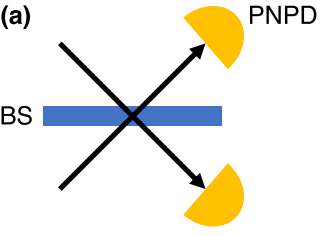

(d)

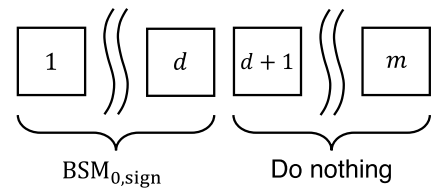

(e)

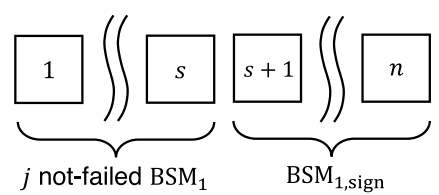

(b)

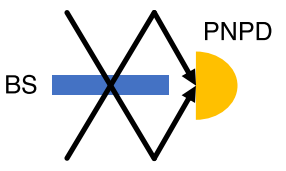

(c)

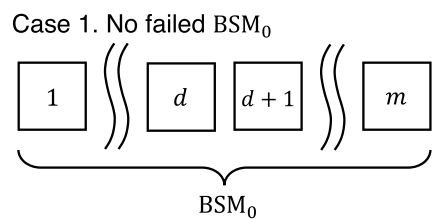

Case 2. $d \leq f$

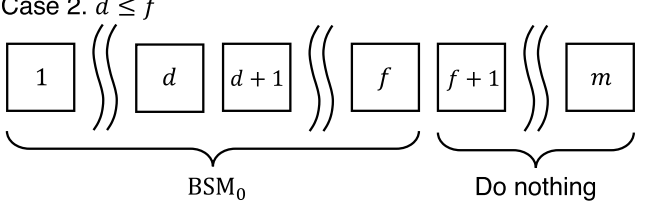

Case 3. $d>f$

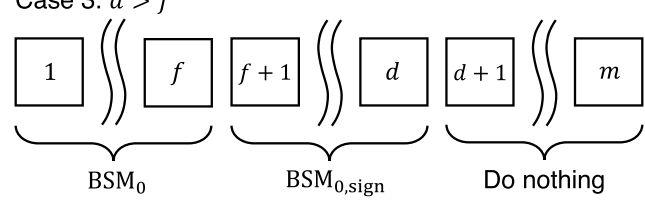

FIG. 4. Overview of the hardware-efficient CBSM scheme. (a) For a BSM $_{0}$ (full physical-level BSM), a 50:50 beam splitter (BS) and two PNPDs are used. (b) For a $\mathrm{BSM}_{0}^{\text {sign }}$ (partial physical-level BSM detecting only the sign), a single PNPD is necessary, instead of two of them. (c) For a $\mathrm{BSM}_{1}$ (full block-level BSM), one of $\mathrm{BSM}_{0}$ and $\mathrm{BSM}_{0}^{\text {sign }}$ is done on each PLS one by one. We define positive integers $d$ and $f$. $d$ is the index of the first PLS such that $\lceil m / 2\rceil$ of the physical-level BSM results until the PLSs have the same sign. $f$ is the index of the first PLS such that the corresponding physical-level BSM fails, which is defined only if such a PLS exists. (CASE 1) If there are no failed BSM ${ }_{0}$ 's (i.e., $f$ is not defined), $\mathrm{BSM}_{0}$ 's are done on the entire PLSs. (CASE 2 ) If $d \leqslant f, \mathrm{BSM}_{0}$ 's are done on the first $f$ PLSs and the remaining PLSs are

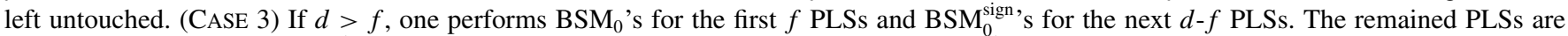
left untouched. (d) For a BSM ${ }_{1}^{\text {sign }}$ (partial block-level BSM detecting only the sign), BSM ${ }_{0}^{\text {sign }}$, s are done for the first $d$ PLSs, and the remaining PLSs are left untouched. (e) For a $\mathrm{BSM}_{2}$ (full logical-level BSM), $\mathrm{BSM}_{1}$ 's are done one by one until $j$ not-failed $\mathrm{BSM}_{1}$ results are obtained, where $j$ is a controllable positive integer referred to as the letter solidity parameter. $\mathrm{BSM}_{1}^{\text {sign }}$, $\mathrm{s}$ are then done for the left blocks.

physical level can be corrected by the majority vote in the block level, thus they do not cause a logical-level $Z$-error with a high probability. Similarly, $X$-errors (letter flips) in the physical level can be corrected by the majority vote in the logical level, thus they also do not cause a logical-level $X$-error with a high probability. Since $Z$-errors are much more common than $X$-errors in the physical level $\left(p_{X} / p_{Z} \lesssim 10^{-3}\right)$, we can infer that the physical-level repetition is crucial for fault-tolerance.

However, we cannot assure that the repetitions always suppress logical errors. Although $Z$-errors can be corrected by the physical-level repetition, the block-level repetition has a rather negative effect on it: Any single remaining $Z$-error among the block-level BSM results can cause a $Z$-error in the logical level. Therefore, a large value of the size of the blocklevel repetition $(n)$ makes the CBSM vulnerable to $Z$-errors. A similar logic applies to $X$-errors: The physical-level repetition has a negative effect on it.

Next, as explained in the previous subsection, a $\mathrm{BSM}_{2}$ fails if all the $\mathrm{BSM}_{1}$ 's fail or the $\mathrm{BSM}_{1}$ results have the same number of both letters, and a $\mathrm{BSM}_{1}$ fails if any single $\mathrm{BSM}_{0}$ fails. The block-level repetition thus suppresses the failure of the CBSM, whereas the physical-level repetition makes it vulnerable to the failure.

In summary, ignoring $X$-errors, which are much more uncommon than $Z$-errors and failures, the physical (block) -level repetition contributes to making the CBSM tolerant to $Z$ errors (failures) but vulnerable to failures ( $Z$-errors). Despite these negative effects, we numerically show in Sec. VI that high success probabilities are still achievable if the survival rate of photons is high enough and the amplitude of the coherent state is large enough.

\section{Improved hardware-efficient CBSM scheme}

In this subsection, we suggest an improved CBSM scheme that is elaborately designed considering hardware efficiency. We explicitly define the cost of a single trial of the CBSM in the last part of this section, but we first regard it as the number of physical-level BSMs required for it.

The unoptimized scheme in Sec. IV A always requires $\mathrm{nm}$ physical-level BSMs, and here we suggest a way to decrease this number. The core idea is that it is redundant to perform full BSMs for all the PLSs or blocks, where the term "full" is used to emphasize that the BSM captures both sign and letter information of the Bell state. For some PLSs or blocks, it is enough to get only the sign information of the Bell state or even do not measure it at all. Especially for the logical level, it is enough to perform full BSMs only for the first few blocks due to the biased noise. The hardware-efficient CBSM scheme presented from now on is summarized in Fig. 4.

\section{Physical level: $\mathrm{BSM}_{0}$ and $\mathrm{BSM}_{0}^{\text {sign }}$}

The $\mathrm{BSM}_{0}$ scheme is completely the same as the scheme given in Sec. II: Using a 50:50 beam splitter and two PNPDs [see Fig. 4(a)], one of the four Bell states can be identified according to the results of the PNPDs, unless the two results are the same (failure). If a $\mathrm{BSM}_{0}$ fails, only the sign of the Bell state can be captured. 
However, we need another ingredient in the physical level for the hardware-efficient CBSM scheme: the partial physicallevel BSM $\left(\mathrm{BSM}_{0}^{\text {sign }}\right)$ identifying only the sign of the physicallevel Bell state [see Fig. 4(b)]. For a $\mathrm{BSM}_{0}^{\text {sign }}$, one needs to measure the parity of $x+y$ in Table I. Therefore, only one PNPD is needed for a $\mathrm{BSM}_{0}^{\text {sign }}$ instead of two of them.

\section{Block level: $\mathrm{BSM}_{1}$ and $\mathrm{BSM}_{1}^{\text {sign }}$}

For a block-level BSM, we perform either $\mathrm{BSM}_{0}$ or $\mathrm{BSM}_{0}^{\text {sign }}$ on each PLS, one by one in order. The process is not parallel, since the determination between $\mathrm{BSM}_{0}$ and $\mathrm{BSM}_{0}^{\text {sign }}$ is affected by the previous measurement results. We first define a positive integer $d \leqslant m$ by the index of the first PLS such that $\lceil m / 2\rceil$ of the physical-level BSM results until that PLS has the same sign. In other words, the result of the majority vote of the signs is definitely determined only by the first $d$ physical-level BSMs, thus the sign information is no longer necessary. Also, we define a positive integer $f \leqslant m$ by the index of the first PLS such that the corresponding BSM fails, which is defined only if such a PLS exists.

Three cases are possible on $\mathrm{BSM}_{0}$ : no failed physicallevel BSMs (i.e., $f$ is not defined), $d \leqslant f$, and $d>f$ [see Fig. 4(c)]. If there are no failed physical-level BSMs (CASE 1 ), it is the same as the unoptimized scheme; $\mathrm{BSM}_{0}$ 's are done for all the PLSs. If $d \leqslant f$ (CASE 2), BSM ${ }_{0}$ 's are done for the first $f$ PLSs. The remained $m-f$ PLSs are left untouched. If $d>f$ (CASE 3), BSM $_{0}$ 's are done for the first $f$ PLSs, then $\mathrm{BSM}_{0}^{\text {sign, }}$ are done for the next $d-f$ PLSs. The remained $m-d$ PLSs are left untouched.

For all three cases, the sign of the block-level Bell state is determined by the signs of the first $d \mathrm{BSM}_{0}$ (or $\mathrm{BSM}_{0}^{\text {sign }}$ ) results. However, its letter is determined only for the first case by the parity of the number of $\mathrm{BSM}_{0}$ results with the letter $\psi$. For the second and third cases, there exists a failed $\mathrm{BSM}_{0}$, thus the number of results with the letter $\psi$ is ambiguous. Hence, the $\mathrm{BSM}_{1}$ fails in these two cases. This is why we can do nothing on the last several PLSs after the sign of the blocklevel BSM is determined.

Like the physical level, we also consider the partial blocklevel BSM $\left(\mathrm{BSM}_{1}^{\text {sign }}\right)$, which determines only the sign of the block-level Bell state [see Fig. 4(d)]. For a BSM ${ }_{1}^{\text {sign }}$, BSM $_{0}^{\text {sign, }}$, are done for the first $d$ PLSs, and the remaining PLSs are left untouched. Defining a positive integer $d \leqslant m$ similarly, the sign of the block-level Bell state is determined by the majority vote of the first $d \mathrm{BSM}_{0}^{\text {sign }}$ results.

\section{Logical level: $\mathrm{BSM}_{2}$}

For a logical-level BSM $\left(\mathrm{BSM}_{2}\right)$ [see Fig. 4(e)], BSM 1 's are done one by one until we get $j$ not-failed results. $j$ is a controllable positive odd integer referred to as the letter solidity parameter in the sense that high values of $j$ lead to high probabilities to get correct letter information. After that, $\mathrm{BSM}_{1}^{\text {sign, }}$, are done for the remaining blocks.

The sign of the resulting logical-level Bell state is determined by the parity of the number of $\mathrm{BSM}_{1}$ or $\mathrm{BSM}_{1}^{\text {sign }}$ results with the minus sign. Its letter is determined by the majority vote of the letters of the $\mathrm{BSM}_{1}$ results. The $\mathrm{BSM}_{2}$ fails if all the $\mathrm{BSM}_{1}$ 's fail or the result of the majority vote is a tie.

Note the difference between $\mathrm{BSM}_{2}$ and $\mathrm{BSM}_{1}$ : The majority vote in $\mathrm{BSM}_{2}$ is taken for a few blocks, while the majority vote in a $\mathrm{BSM}_{1}$ is taken for all the PLSs. This asymmetry comes from the fact that the noise is strongly biased; in $\mathrm{BSM}_{0}$, $X$-errors are much less likely to occur compared to $Z$-errors, as shown in Fig. 2.

\section{Calculation of the cost}

At the beginning of this subsection, we determine the cost of a CBSM by the number of physical-level BSMs used for the measurement. However, considering that PNPDs are the most difficult elements when implementing the $\mathrm{BSM}_{0}$ scheme and a $\mathrm{BSM}_{0}^{\text {sign }}$ uses one PNPD while a $\mathrm{BSM}_{0}$ uses two of them, it is reasonable to assign each $\mathrm{BSM}_{0}^{\text {sign }}$ half the cost of one $\mathrm{BSM}_{0}$.

Definition 2. The cost function $C$ of a CBSM is defined by

$$
C:=N_{\mathrm{BSM}_{0}}+\frac{1}{2} N_{\mathrm{BSM}_{0}^{\mathrm{sign}}},
$$

where $N_{\mathrm{BSM}_{0}}$ and $N_{\mathrm{BSM}_{0}^{\text {sign }}}$ are the numbers of $\mathrm{BSM}_{0}$ 's and $\mathrm{BSM}_{0}^{\text {sign, }}$ s used for the CBSM, respectively. We also define the expected cost $C_{\exp }(n, m, \alpha, j ; \eta)$ by the expectation value of the cost $C$ for the CBSM scheme specified by the parameters $(n, m, \alpha, j)$ and the photon survival rate $\eta$, under the assumption that the initial state before suffering photon loss is one of the four logical Bell states with equal probabilities.

We use the expected cost $C_{\exp }$ as a measure of the hardware-efficiency of a CBSM scheme. It is straightforward to see that the CBSM scheme in the previous subsection has a lower expected cost than the unoptimized one in Sec. IV A. Not only that, it is designed to minimize the expected cost. For a $\mathrm{BSM}_{1}$, the numbers of $\mathrm{BSM}_{0}$ 's and $\mathrm{BSM}_{0}^{\text {sign, }} \mathrm{s}$ are minimized while keeping the result the same as that of the corresponding $\mathrm{BSM}_{1}$ in the unoptimized scheme. For $\mathrm{BSM}_{2}$, the expected cost is determined by the controllable letter solidity parameter $j$.

\section{Parallelization of the concatenated Bell-state measurement}

The two CBSM schemes in Secs. IV A and IVC are processed in a completely or partially distributed manner, which makes efficient information processing possible by parallelization. The unoptimized scheme is done in a completely distributed manner, i.e., a $\mathrm{BSM}_{2}$ is split by $2 \mathrm{~nm} \mathrm{BSM}_{0}$ 's, each of which is performed independently. The $\mathrm{BSM}_{0}$ results are collected classically to deduct the logical-level BSM result.

On the other hand, the hardware-efficient scheme can be done in a partially distributed manner allowing partial parallelization, with requirements of classical communication channels between different PLSs and blocks. In a $\mathrm{BSM}_{2}$, $\mathrm{BSM}_{1}$ 's can be done parallelly for the first $j$ blocks, then one by one until obtaining $j$ not-failed $\mathrm{BSM}_{1}$ results, where $j$ is the letter solidity parameter. $\mathrm{BSM}_{1}^{\text {sign, }}$ s for the remaining blocks can also be done parallelly. In a BSM $1, \mathrm{BSM}_{0}$ 's should be done one by one until a $\mathrm{BSM}_{0}$ fails, so $\mathrm{BSM}_{0}$ 's in all three cases cannot be done parallelly. CASE 3 can be partially parallelized only if $f<m / 2$ : $\mathrm{BSM}_{0}^{\text {sign, }}$, can be done parallelly for $(f+1)$ th to $\lceil m / 2\rceil$ th PLS since $d$ is always larger than 
$m / 2$. In $\mathbf{B S M}_{1}^{\text {sign }}, \mathbf{B S M}_{0}^{\text {sign }}$ can be done parallelly for the first $\lceil m / 2\rceil$ PLSs, then one by one for the remaining PLSs.

Therefore, the hardware efficiency is the result of the sacrifice of parallelization. We can still widen the range of parallelization by adjusting the scheme appropriately at the expense of reducing hardware efficiency. For example, in a $\mathrm{BSM}_{2}, \mathrm{BSM}_{1}$ 's can be done for the first $j$ blocks, not for the first not-failed $j$ blocks. Moreover, in a $\mathrm{BSM}_{1}$ and $\mathrm{BSM}_{1}^{\text {sign }}$, instead of determining the type of the BSM $\left(\mathrm{BSM}_{0}\right.$ or $\mathrm{BSM}_{0}^{\text {sign }}$ ) separately for each PLS, we can divide the PLSs into several groups and perform BSMs of the same type parallelly on PLSs in each group. However, we use the original hardware-efficient CBSM scheme for the numerical simulation in Sec. VI to figure out the best possible performance.

\section{PROBABILITY DISTRIBUTIONS OF CONCATENATED BELL-STATE MEASUREMENT RESULTS}

In this section, we present the analytic expressions of the probability distributions of CBSM results conditioning to the initial Bell state before suffering photon loss. We only consider the unoptimized CBSM scheme since the measurement results of the hardware-efficient CBSM scheme are the direct consequences of those of the unoptimized scheme. Here, we show only the final results. A brief outline for inducing the results is presented in Appendix C.

The results of this section have two important meanings: First, the probability distributions are written in simple matrix-form expressions, which makes it possible to sample arbitrary CBSM results at a high rate since a matrix calculation can be done much faster on a computer compared to calculating the same thing by simple loops. Second, the results can be easily generalized to any CBSM schemes with other encoding methods such as multiphoton polarization encoding [11].

\section{A. Probability distributions of block-level results}

We first find the probability distributions of block-level BSM results, conditioning to the initial block-level Bell state. A single BSM $_{1}$ result can be expressed by two vectors, $\mathbf{x}, \mathbf{y} \in$ $\{0,1,2,3\}^{m}$, where their $i$ th elements are the two PNPD results of the $i$ th $\mathrm{BSM}_{0}$. What we want is the conditional probability $\operatorname{Pr}\left(\mathbf{x}, \mathbf{y} \mid B_{1}\right)$ for each $\left|B_{1}\right\rangle \in \mathcal{B}_{1}:=\left\{\left|\phi_{ \pm}^{(m)}\right\rangle,\left|\psi_{ \pm}^{(m)}\right\rangle\right\}$.

First, we define a $4 \times 4$ matrix $\tilde{\mathbf{M}}_{x, y}^{ \pm}$for each pair of $x, y \in$ $\{0,1,2,3\}$ as

$$
\tilde{\mathbf{M}}_{x, y}^{ \pm}:=\left(\begin{array}{cccc}
M_{11}^{ \pm} & M_{12}^{ \pm} & M_{12}^{ \pm} & M_{22}^{ \pm} \\
M_{12}^{ \pm} & M_{11}^{ \pm} & M_{22}^{ \pm} & M_{12}^{ \pm} \\
M_{12}^{ \pm} & M_{22}^{ \pm} & M_{11}^{ \pm} & M_{12}^{ \pm} \\
M_{22}^{ \pm} & M_{12}^{ \pm} & M_{12}^{ \pm} & M_{11}^{ \pm}
\end{array}\right),
$$

where

$$
\begin{aligned}
& M_{11}^{ \pm}:=\left\langle\phi_{ \pm}\left|M_{x, y}\right| \phi_{ \pm}\right\rangle, \\
& M_{12}^{ \pm}:=\left\langle\phi_{ \pm}\left|M_{x, y}\right| \psi_{ \pm}\right\rangle, \\
& M_{22}^{ \pm}:=\left\langle\psi_{ \pm}\left|M_{x, y}\right| \psi_{ \pm}\right\rangle
\end{aligned}
$$

are the matrix elements of the POVM elements of the $\mathrm{BSM}_{0}$ and they can be calculated from Eqs. (A2) in Appendix A. De- noting the $k$ th elements of $\mathbf{x}$ and $\mathbf{y}$ by $x_{k}$ and $y_{k}$, respectively, we get

$$
\begin{aligned}
& \operatorname{Pr}\left(\mathbf{x}, \mathbf{y} \mid \phi_{ \pm}^{(m)}\right)=\frac{1}{2} \tilde{N}_{ \pm}(1, m)^{2} v_{m 1}^{ \pm}(\mathbf{x}, \mathbf{y}), \\
& \operatorname{Pr}\left(\mathbf{x}, \mathbf{y} \mid \psi_{ \pm}^{(m)}\right)=\frac{1}{2} \tilde{N}_{ \pm}(1, m)^{2} v_{m 4}^{ \pm}(\mathbf{x}, \mathbf{y}),
\end{aligned}
$$

where $\tilde{N}_{ \pm}(1, m)$ is defined in Eq. (9) and $v_{m \mu}^{ \pm}(\mathbf{x}, \mathbf{y})$ is the $\mu$ th element of a four-dimensional vector $\mathbf{v}_{m}^{ \pm}(\mathbf{x}, \mathbf{y})=$ $\tilde{\mathbf{M}}_{x_{m}, y_{m}}^{ \pm} \cdots \tilde{\mathbf{M}}_{x_{1}, y_{1}}^{ \pm}(1,0,0,0)^{T}$.

$\mathrm{A}$ brief outline for inducing these results is presented in Appendix B 1.

\section{B. Probability distributions of logical-level results}

Now, we consider the probability distributions of logicallevel results conditioning to the initial logical-level Bell state, which is the goal of this section. A single CBSM result can be expressed by two matrices $\mathbf{X}, \mathbf{Y} \in\{0,1,2,3\}^{n \times m}$, where their $(i, k)$ elements are the two PNPD results of the $k$ th PLS of the $i$ th block. What we want is the conditional probability $\operatorname{Pr}\left(\mathbf{X}, \mathbf{Y} \mid B_{2}\right)$ for each $\left|B_{2}\right\rangle \in \mathcal{B}_{2}:=\left\{\left|\Phi_{ \pm}\right\rangle,\left|\Psi_{ \pm}\right\rangle\right\}$.

We first define $2 \times 2$ matrices $\tilde{\mathbf{L}}_{\mathbf{x}, \mathbf{y}}^{\phi}$ and $\tilde{\mathbf{L}}_{\mathbf{x}, \mathbf{y}}^{\psi}$ for each pair of $\mathbf{x}, \mathbf{y} \in\{0,1,2,3\}^{m}$ in a similar way to the block-level case:

$$
\tilde{\mathbf{L}}_{\mathbf{x}, \mathbf{y}}^{\phi()}:=\left(\begin{array}{cc}
L_{+}^{\phi(\psi)} & L_{-}^{\phi(\psi)} \\
L_{-}^{\phi(\psi)} & L_{+}^{\phi(\psi)}
\end{array}\right),
$$

where

$$
L_{ \pm}^{\phi(\psi)}:=\left[1 \pm u(\alpha, m)^{2}\right]\left\langle\phi_{ \pm}^{(m)}\left(\psi_{ \pm}^{(m)}\right)\left|\bigotimes_{k=1}^{m} M_{x_{k}, y_{k}}\right| \phi_{ \pm}^{(m)}\left(\psi_{ \pm}^{(m)}\right)\right\rangle,
$$

$u(\alpha, m)$ is defined in Eq. (11), and $x_{k}, y_{k}$ are the $k$ th elements of $\mathbf{x}$ and $\mathbf{y}$, respectively. Note that the right-hand side of Eq. (15) can be calculated from Eqs. (14). Denoting the $i$ th row vectors of $\mathbf{X}$ and $\mathbf{Y}$ by $\mathbf{x}_{i}$ and $\mathbf{y}_{i}$, respectively, we get

$$
\begin{aligned}
& \operatorname{Pr}\left(\mathbf{X}, \mathbf{Y} \mid \Phi_{+}\left(\Psi_{+}\right)\right)=\tilde{N}_{+}(n, m)^{2} w_{n 1}^{\phi(\psi)}(\mathbf{X}, \mathbf{Y}), \\
& \operatorname{Pr}\left(\mathbf{X}, \mathbf{Y} \mid \Phi_{-}\left(\Psi_{-}\right)\right)=\tilde{N}_{-}(n, m)^{2} w_{n 2}^{\phi(\psi)}(\mathbf{X}, \mathbf{Y}),
\end{aligned}
$$

where $\tilde{N}_{ \pm}(n, m)$ is defined in Eq. (9) and $w_{n \mu}^{\phi(\psi)}(\mathbf{X}, \mathbf{Y})$ is the $\mu$ th element of the two-dimensional vector $\mathbf{w}_{n}^{\phi(\psi)}(\mathbf{X}, \mathbf{Y}):=$ $\tilde{\mathbf{L}}_{\mathbf{x}_{n}, \mathbf{y}_{n}}^{\phi(\psi)} \cdots \tilde{\mathbf{L}}_{\mathbf{x}_{1}, \mathbf{y}_{1}}^{\phi(\psi)}(1,0)^{T}$. A brief outline for inducing these results is presented in Appendix B 2.

In conclusion, one can calculate the probability distributions of CBSM results by systematic matrix operations as described above. The probability distributions can then be used to sample the CBSM results for numerical calculations.

\section{NUMERICAL CALCULATIONS}

In this section, we show the results of the numerical calculations. We use the Monte Carlo method for the simulation: sampling the measurement results randomly and counting the number of successes, errors, and failures. We sample the result of each physical-level BSM one by one in order, which is exponentially faster than sampling all the measurement results at once. The detailed method for sampling the CBSM results using the results in the previous section is presented in Appendix C. 
$p_{l}$

(a)

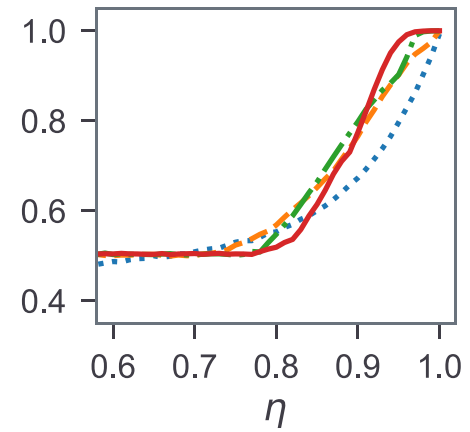

$\eta$ (b)

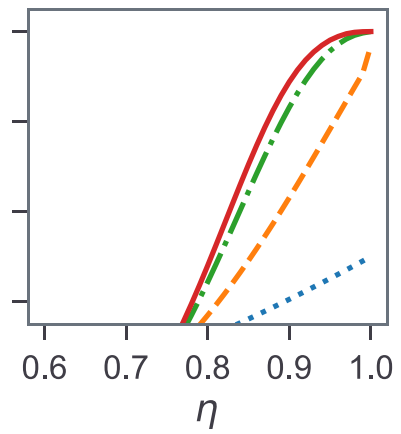

$$
\begin{array}{ll}
\ldots . . & \text { No repetition } \quad-\cdot 11<C_{\exp } \leq 15 \\
---1<C_{\exp } \leq 5-21<C_{\exp } \leq 25
\end{array}
$$

FIG. 5. Success probabilities $p_{I}$ of a CBSM with (a) coherentstate qubits or (b) multiphoton polarization qubits [11] against the photon survival rate $\eta$ for different ranges of the expected cost $C_{\exp }$. For coherent-state qubits, the amplitude $\alpha$ is fixed to $\alpha=1.6$ and the letter solidity parameter $j$ is chosen to maximize $p_{I}$ for each $\eta$ and range of $C_{\text {exp }}$. For polarization qubits, we define $C_{\text {exp }}:=n m$, which is the number of physical-level BSMs used for one CBSM. Part (a) shows that the repetition indeed contributes to enhance the success probability. Comparing (a) and (b), we can see that the CBSM with coherent-state qubits outperforms that with polarization qubits when the repetition size is relatively small.

Note that there are four free parameters related to the hardware-efficient CBSM scheme: $n, m, \alpha$, and $j . n$ and $m$ determine the block- and physical-level repetition sizes of the scheme, respectively. $\alpha$ is the amplitude of the coherent state constituting the logical basis. $j$ is the letter solidity parameter, which is the number of not-failed blocks used for the majority vote of letters in the logical level.

\section{A. Performance analysis}

Now, we analyze the performance of the hardware-efficient CBSM scheme suggested in Sec. IV C by calculating numerically the success, error, and failure probabilities of the scheme with various settings of the parameters $(n, m, \alpha, j)$. For the simulation, we assume that both systems have the same photon survival rates $\eta$. We use the Monte Carlo method as mentioned above. For each trial, we first choose one of the four Bell states as the initial state with equal probabilities, sample the physical-level BSM results for the selected initial state, then determine the logical Bell state using the hardwareefficient CBSM scheme. Repeating the process enough times, we determine the success, $Z$-error, and failure probabilities (referred to as $p_{I}, p_{Z}$, and $p_{\text {fail }}$, respectively) of the CBSM scheme. We also calculate the expected cost $C_{\exp }$ defined in Definition 2.

Figure 5 illustrates $p_{I}$ of a CBSM with coherent-state qubits or polarization qubits [11] against the photon survival rate $\eta$ for different ranges of $C_{\mathrm{exp}}$, where $p_{I}$ is maximized for each $\eta$ and $C_{\text {exp. }}$. Figure 5(a) shows that the repetition indeed enhances the performance if $\eta \gtrsim 0.8$ compared to the case without repetition. The effect of the repetition is especially crucial if $\eta$ is close to unity. For example, if $\eta=0.95, p_{I}=$
0.80 without repetition, but it reaches 0.90 with just a little repetition $\left(C_{\exp } \leqslant 5\right)$, and up to 0.99 for $31<C_{\exp } \leqslant 35$. In other words, it is clear evidence that high success rates close to unity are achievable by the CBSM if the photon survival rate is sufficiently high. Additionally, comparing Figs. 5(a) and 5(b), we can see that the CBSM with coherent-state qubits outperforms that with multiphoton polarization qubits when the repetition size is relatively small $\left(C_{\exp } \leqslant 5\right)$. For instance, if $\eta=0.99$, the CBSM with coherent-state qubits achieves $p_{I}=0.90$ for $C_{\exp } \leqslant 5$, while that with multiphoton polarization qubits reaches only $p_{I}=0.78$.

In Figs. 6(a)-6(c), we compare $p_{I}, p_{Z}$, and $p_{\text {fail }}$ against $n$ and $m$ for two different values of $\alpha$ (1.2 and 1.6) while fixing $\eta=0.99$. From this figure, we can check the dependence of $p_{Z}$ and $p_{\text {fail }}$ on the repetition: The physical-level repetition $(m>1)$ suppresses $Z$-errors and the block-level repetition $(n>1)$ suppresses failures, as argued in Sec. IV B. Moreover, the negative effects discussed in Sec. IV B that the physical (block) -level repetition makes the CBSM vulnerable to failures ( $Z$-errors) are also shown in the figure, and despite them, a success probability close to unity still can be achieved.

We additionally plot the approximate average teleportation fidelity $F_{\text {tel }}$ while fixing $\eta=0.99$ in Fig. 6(d), showing that $F_{\text {tel }}$ near unity is achievable even with small repetition sizes. It is another widely used measure for evaluating the performance of a BSM scheme defined as follows: Considering the standard teleportation scheme [6] with the BSM scheme, let $\left|\psi_{\text {in }}\right\rangle$ and $\rho_{\text {out }}$ be the pure input state and the corresponding mixed output state, respectively. The average teleportation fidelity is defined as $F_{\text {tel }}:=\overline{\left\langle\psi_{\text {in }}\left|\rho_{\text {out }}\right| \psi_{\text {in }}\right\rangle}$, where the averaging is taken on the Bloch sphere in the logical basis:

$$
\overline{f\left(\left|\psi_{\mathrm{in}}\right\rangle\right)}:=\frac{1}{4 \pi} \int_{0}^{\pi} d \theta \sin \theta \int_{0}^{2 \pi} d \phi f\left(\left|\psi_{\mathrm{in}}\right\rangle\right)
$$

for $\quad\left|\psi_{\text {in }}\right\rangle=N\left(\cos \frac{\theta}{2}\left|0_{L}\right\rangle+e^{i \phi} \sin \frac{\theta}{2}\left|1_{L}\right\rangle\right) \quad$ with $\quad N:=$ $\left(1+\sin \theta \cos \phi\left\langle 0_{L} \mid 1_{L}\right\rangle\right)^{-1 / 2}$. It requires huge computational resources to calculate $F_{\text {tel }}$ exactly since we need to know all the off-diagonal terms of the POVM elements of the CBSM in the basis of the logical-level Bell states as shown in Eq. (D2), which cannot be done with the Monte Carlo method unlike the diagonal terms. Nevertheless, if the two systems have the same photon survival rate, we can approximate $F_{\text {tel }}$ with the following simple expression derived in Appendix D:

$$
F_{\text {tel }}=p_{I}+\frac{p_{Z}}{2}+O\left(\left(m e^{-2 \eta|\alpha|^{2}}\right)^{n}\right) .
$$

Lastly, in Fig. 7, $p_{I}$ is plotted against $\alpha$ and $C_{\text {exp }}$ for four different survival rates $(\eta=1,0.99,0.95$, and 0.9$)$. The figure shows that $p_{I}>0.98$ can be reached for $\eta \geqslant 0.95$ and appropriate values of $\alpha$ if sufficiently large costs of the CBSM are available. In lossless cases $(\eta=1)$, the success probability reaches very close to unity for any $\alpha \gtrsim 0.4$ with just a little repetition. As the photon survival rate gets smaller, appropriately large values of $\alpha$ and the cost are required to reach high success probabilities. In detail, to reach $p_{I}>0.98$, we need $\alpha \gtrsim 0.8$ for $\eta=0.99$ and $\alpha \gtrsim 1.4$ for $\eta=0.95$. Nevertheless, the figure also indicates that a higher value of $\alpha$ does not always guarantee a higher success rate due to dephasing by photon loss, which is especially evident when $\eta=0.95$. 
(a)
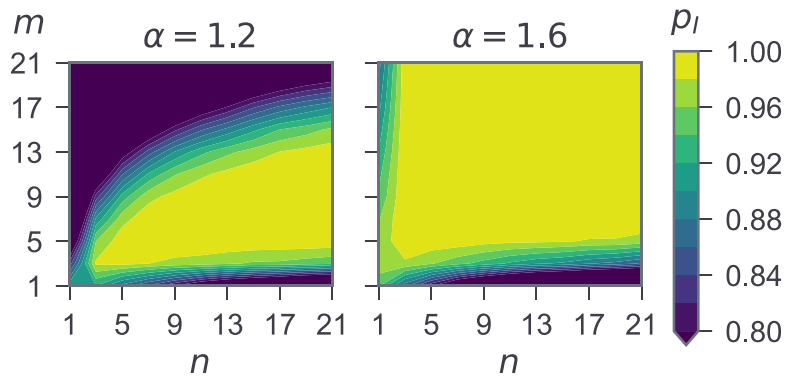

(b)
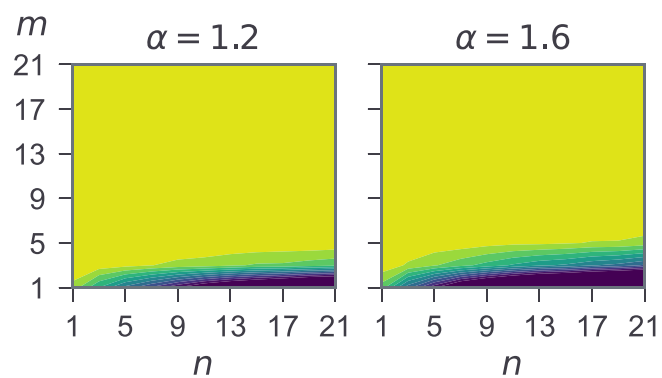

$p_{Z}$

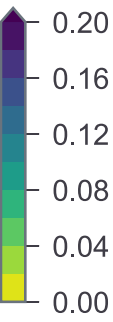

(c)
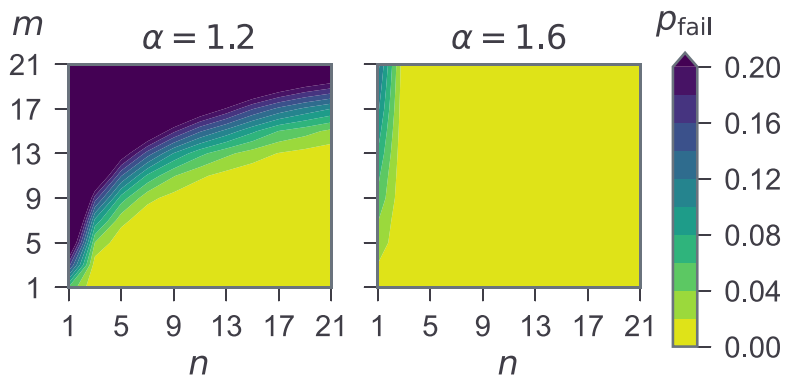

(d)
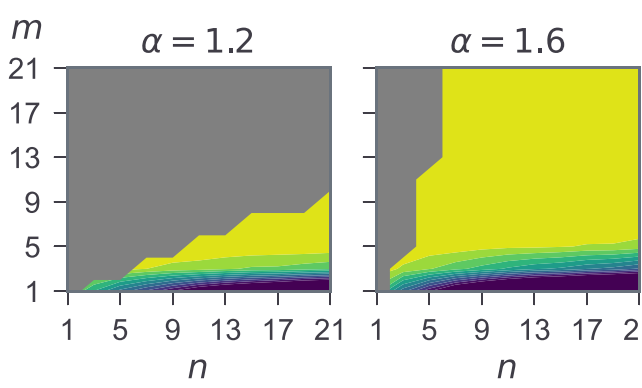

$F_{\text {tel }}$

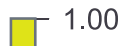

0.98

0.96

0.94

0.92

0.90

FIG. 6. (a) Success, (b) $Z$-error, and (c) failure probabilities $p_{I}$, $p_{Z}, p_{\text {fail }}$ of CBSM, and (d) the corresponding average teleportation fidelity $F_{\text {tel }}$ against the repetition sizes $n$ and $m$ for the two values of coherent-state amplitudes $\alpha=1.2$ and 1.6. The photon survival rate $\eta$ is fixed to 0.99 , and $j$ is selected to maximize $p_{I}$ for each $(n, m)$ point. It clearly shows that physical-level repetition suppresses $Z$ error and block-level repetition suppress failure. $F_{\text {tel }}$ is approximately calculated using Eq. (17). Gray areas in (d) indicate the regions of $\left(m e^{-2 \eta|\alpha|^{2}}\right)^{n}>0.01\left(1-F_{\text {tel }}\right)$ where the approximation may be wrong.

\section{B. Quantum repeater with concatenated Bell-state measurement}

In this subsection, we investigate the performance of the quantum repeater scheme using the proposed CBSM scheme for quantum error correction as one of the key applications of the BSM.

\section{Network design}

We follow the network design in Ref. [11], which suggests an all-optical quantum network with a quantum repeater exploiting the CBSM scheme with multiphoton polarization qubits. As shown in Fig. 8(a), we consider one-way quantum communication with which a qubit encoded with the modified parity encoding is transmitted to the other end. While traveling between two ends with a total distance of $L$, the qubit passes through multiple repeater stations separated by intervals of $L_{0}$. Figure 8(b) illustrates the processes inside each repeater station. In each of them, a Bell state $\left|\Phi_{+}\right\rangle$is prepared and a CBSM is performed jointly on the incoming qubit and one side of the Bell state. The quantum information in the incoming qubit is then teleported to the other side of the Bell state, which is transmitted to the next station. The measurement result of the CBSM in each station is sent classically to the final end for recovering the original quantum information. Because of the fault tolerance of the CBSM scheme, each repeater station can correct possible logical errors that originated from photon loss, which makes a long-range transmission of quantum information possible.

We assume the two sources of photon loss: internal loss in each repeater station and loss during transmission between stations with survival rates of $\eta_{0}$ and $\eta_{L_{0}}:=e^{-L_{0} / L_{\text {att }}}$, respectively, where $L_{\text {att }}=22 \mathrm{~km}$ is the attenuation length. Therefore, the survival rates of two systems on which CBSM is jointly performed is $\eta_{1}:=\eta_{0} e^{-L_{0} / L_{\text {att }}}$ and $\eta_{2}:=\eta_{0}$.

\section{Quantification of the performance}

One way to quantify the performance of a quantum repeater scheme is the asymptotic key generation rate $R$ of quantum key distribution (QKD), which is the expected length of a fully secure key that can be produced per unit time $[44,62]$. More precisely, it is the product of the raw-key rate (namely, the length of a raw key that can be produced per unit time) and the secret fraction (namely, the fraction of the length of a fully secure key to the length of a raw key in the asymptotic case of $N \rightarrow \infty$, where $N$ is the number of signals) [62]. We use $R t_{0}$ as the measure of performance, where $t_{0}$ is the time taken in one repeater station, which we call the expected key length. The expected key length is given by [44]

$$
R t_{0}=\max \left[P_{s}\{1-2 h(Q)\}, 0\right],
$$

where $P_{s}$ is the probability not to fail during the entire transmission, $Q$ is the average quantum bit error rate (QBER), and $h(Q):=-Q \log _{2}(Q)-(1-Q) \log _{2}(1-Q)$ is the binary entropy function. The probability $P_{s}$ is given by

$$
P_{s}=\left(1-p_{\text {fail }}\right)^{L / L_{0}},
$$

where $p_{\text {fail }}$ is the failure probability of a CBSM in a single repeater station. The average QBER $Q$ is defined by $Q=\left(Q_{X}+Q_{X}\right) / 2$, where $Q_{X}$ and $Q_{Z}$ are given as

$$
Q_{X / Z}=\frac{1}{2}\left[1-\left(\frac{p_{I} \mp p_{X} \pm p_{Z}-p_{Y}}{p_{I}+p_{X}+p_{Z}+p_{Y}}\right)^{L / L_{0}}\right],
$$

where $p_{I}, p_{X}, p_{Y}$, and $p_{Z}$ are the success, $X$-error, $Y$-error, and $Z$-error probabilities of a CBSM in a single repeater station, respectively. 

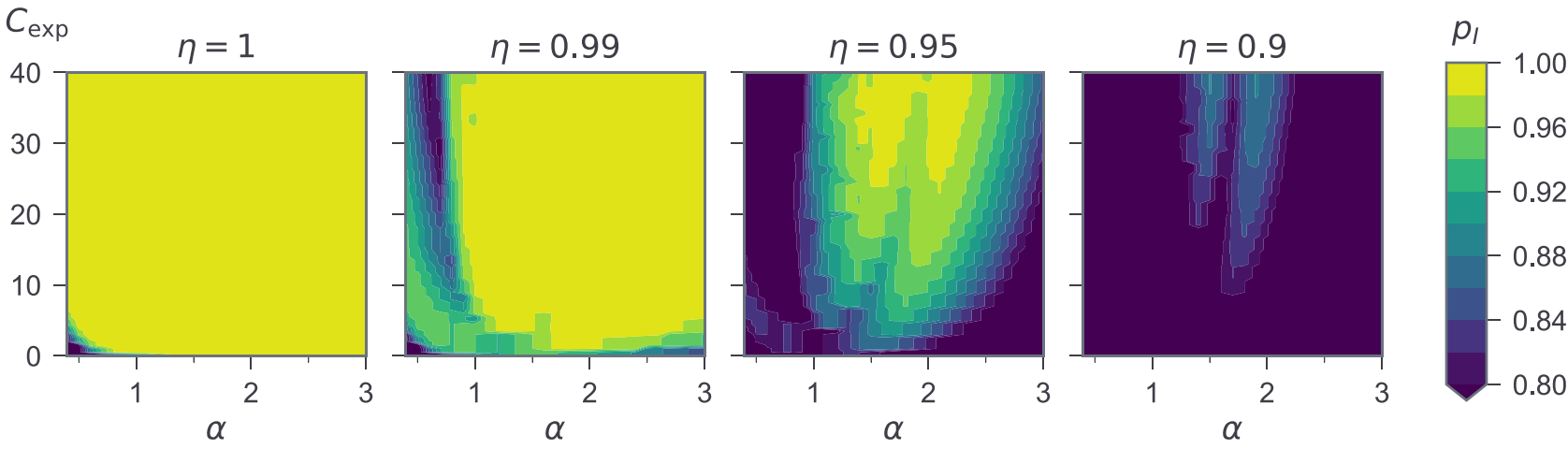

FIG. 7. Success probabilities $p_{I}$ against the coherent-state amplitude $\alpha$ and the expected $\operatorname{cost} C_{\exp }$ for the different values of the photon survival rate $(\eta)$ of both parties. $p_{I}$ is selected by $\max \left\{p_{I}\left(n, m, \alpha_{0}, j\right) \mid C_{\exp }\left(n, m, \alpha_{0}, j ; \eta\right) \in\left[C_{0}-2, C_{0}+2\right)\right\}$ for each point $\left(\alpha_{0}, C_{0}\right)$. The figure indicates that a large value of $\alpha$ does not always guarantee a high success probability, which is especially evident when $\eta=0.95$.

We also define the effective total cost $Q_{\text {tot }}$ of the quantum repeater by

$$
Q_{\mathrm{tot}}:=\frac{C_{\mathrm{exp}}}{R t_{0}} \times \frac{L}{L_{0}},
$$

where $C_{\text {exp }}$ is the expected cost of a CBSM in a single repeater station defined in Definition 2. $Q_{\text {tot }}$ quantifies the expected total cost of CBSMs to generate a secret key with unit length. In the numerical calculations, we try to find the set of parameters $(n, m, \alpha, j)$ and station interval $L_{0}$ which minimizes $Q_{\text {tot }}$.

\section{Results}

We find the optimal parameter sets minimizing the effective total cost $Q_{\text {tot }}$ for the total distance $L$ of 1000 and $10000 \mathrm{~km}$ while fixing $\eta_{0}=0.99$. The parameter sets together with the corresponding effective total costs $Q_{\text {tot }}$ and expected key lengths $R t_{0}$ are calculated as

$$
\begin{aligned}
L=1000 \mathrm{~km}: & \\
(n, m, \alpha, j) & =(3,31,1.9,1), L_{0}=0.7 \mathrm{~km} \\
\rightarrow Q_{\mathrm{tot}} & =(1.019 \pm 0.003) \times 10^{5}, \\
R t_{0} & =0.71 \pm 0.02,
\end{aligned}
$$

$$
\begin{aligned}
L=10000 \mathrm{~km}: & \\
(n, m, \alpha, j) & =(5,41,1.8,3), L_{0}=0.9 \mathrm{~km} \\
\rightarrow Q_{\mathrm{tot}} & =(2.09 \pm 0.05) \times 10^{6} \\
R t_{0} & =0.78 \pm 0.02 .
\end{aligned}
$$

Figure 9 shows $Q_{\text {tot }}$ and $R t_{0}$ of the quantum repeater against the repetition sizes $n$ and $m$ when $L=1000 \mathrm{~km}$ for different settings of the coherent-state amplitude $\alpha$. Here, $\alpha, L_{0}$, and the letter solidity parameter $j$ are selected to minimize $Q_{\text {tot }}$ or maximize $R t_{0}$ if they are not fixed explicitly. Figure 9(b) indicates that $R t_{0}$ arbitrarily close to unity can be obtained for sufficiently large values of $n$ and $m$. In particular, $m$ should be large enough to fix $Z$-errors. On the other hand, since $X$-errors are very rare compared to failures and $Z$-errors, $n$ does not need to be very large, although it should be larger than 1 to suppress failures.

Comparing the results for $\alpha=1.4$ and 1.9 in Fig. 9, the CBSM with a small value of $\alpha$ requires a relatively large value of $n$ to reach low $Q_{\text {tot }}$ and high $R t_{0}$. This is due to the fact that the CBSM becomes more vulnerable to failures as $\alpha$ decreases and the effect of failures can be mitigated by increasing $n$ as discussed in Sec. IV B. Additionally, the minimal attainable (a)

(b)

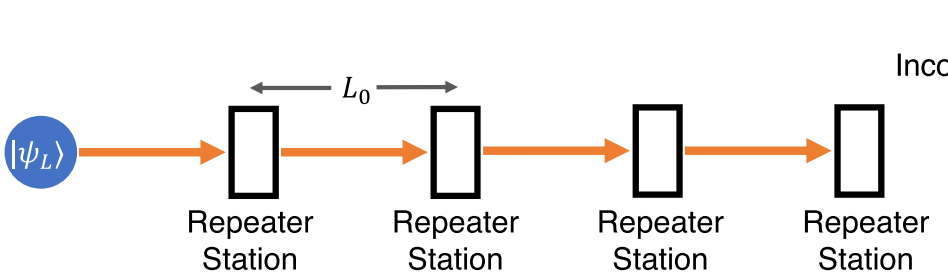

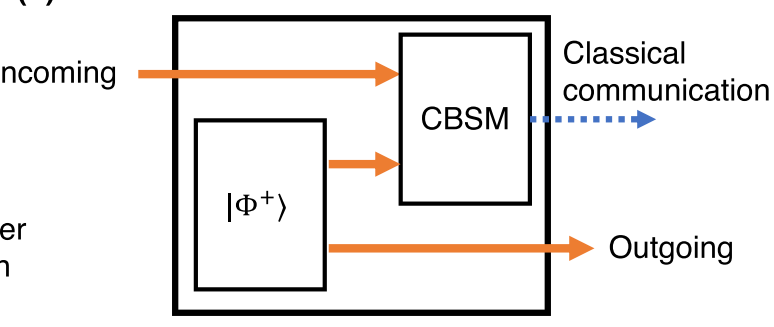

Repeater Station

FIG. 8. (a) Schematic of quantum information transmission through the quantum repeater scheme. Quantum information encoded in modified parity encoding is transmitted to the other end. It passes through multiple repeater stations where the interval is $L_{0}$. (b) Schematic of the process inside each repeater station. A Bell state $\left|\Phi^{+}\right\rangle$is prepared inside the station, and a CBSM is performed between the incoming qubit and one side of the Bell state. The quantum information inside the incoming qubit is then teleported to the other side of the Bell state, which is then transmitted to the next repeater station. The measurement result of the CBSM is sent classically to the final end to recover the original quantum information. Due to the fault tolerance of the CBSM scheme, each repeater station can correct possible logical errors from photon loss that the incoming qubit suffers. 
(a) $m$

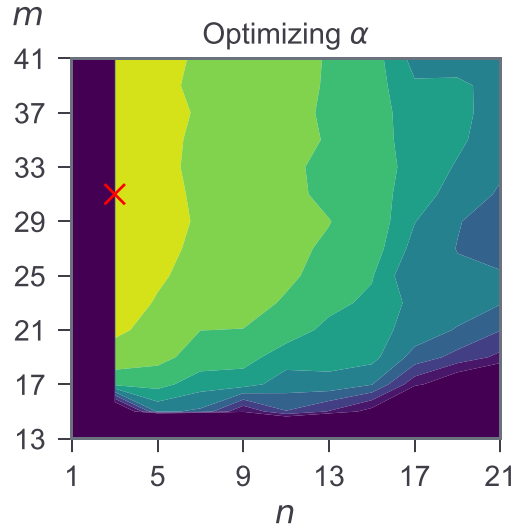

(b)

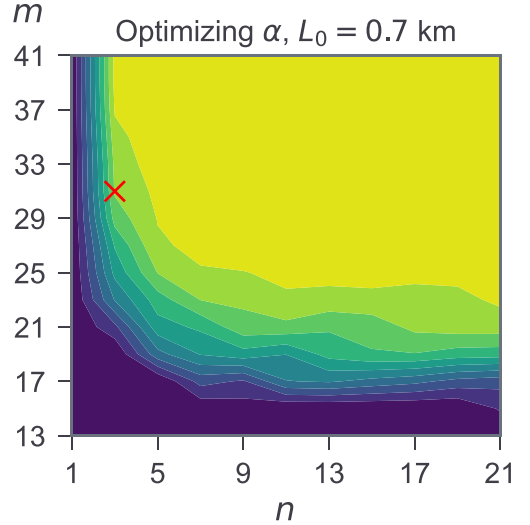

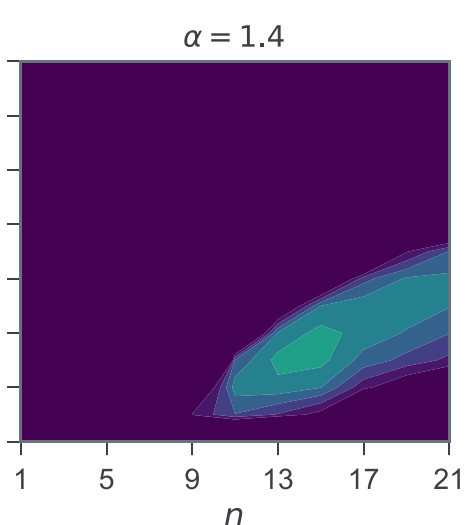

$n$
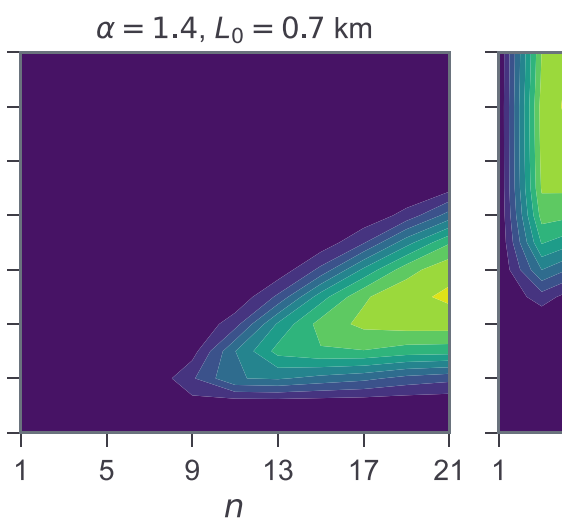

$\alpha=1.9, L_{0}=0.7 \mathrm{~km}$

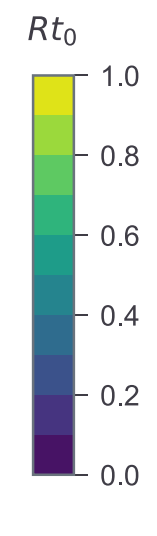

FIG. 9. (a) The effective total cost $Q_{\text {tot }}$ and (b) the expected key length $R t_{0}$ of the quantum repeater against the repetition sizes $n$ and $m$ for three different settings of the coherent-state amplitude $\alpha$ : optimizing $\alpha$, fixing $\alpha=1.4$, and fixing $\alpha=1.9$. We fix the total distance as $L=1000 \mathrm{~km}$ and the internal photon survival rate in each station as $\eta_{0}=0.99$. To calculate $R t_{0}$, we also fix the station interval $L_{0}=0.7 \mathrm{~km}$. For each $(n, m)$ point, other parameters such as the letter solidity parameter $j$ and the station interval $L_{0}$ (only for $Q_{\text {tot }}$ ) are selected to minimize $Q_{\text {tot }}$ or maximize $R t_{0}$. The red X marks indicate the optimal point where $Q_{\text {tot }}$ is minimized. The parameters at this point are $(n, m, \alpha, j)=$ $(3,31,1.9,1)$ and $L_{0}=0.7 \mathrm{~km} . R t_{0}=0.71 \pm 0.02$ and $Q_{\text {tot }}=(1.019 \pm 0.003) \times 10^{5}$ at this point, where the range is the $95 \%$ confidence interval.

$Q_{\text {tot }}$ is smaller for $\alpha=1.9$ than for $\alpha=1.4$. The dependence of the performance of the repeater network on $\alpha$ is more clearly shown in Fig. 10(a), where $Q_{\text {tot }}$ and the corresponding value of $R t_{0}$ are plotted against $\alpha$ while fixing $L=1000 \mathrm{~km}$. Here, $Q_{\text {tot }}$ is minimal at $\alpha=1.9$, which indicates that the parity code with $\alpha>2$ that is hard to generate is unnecessary to attain an efficient repeater.

We also plot the dependence of the optimal $Q_{\text {tot }}$ and the corresponding $R t_{0}$ to the station interval $L_{0}$ in Fig. 10(b). It shows that $Q_{\text {tot }}$ is minimal when $L_{0}$ is around $0.6-1.0 \mathrm{~km}$.

Our repeater scheme shows a similar scale of performance to that of the CBSM based on the multiphoton polarization qubit [11], where optimal $Q_{\mathrm{tot}}=6.5 \times 10^{4}$ and the corresponding key generation rate is 0.70 obtained for $L=1000 \mathrm{~km}$ and $\eta_{0}=0.99$ [63], although a strictly fair comparison is impossible due to the differences in the physical-level BSM schemes. Even though we cannot say our repeater scheme is better than that in Ref. [11], it is still a remarkable result considering that the scheme in Ref. [11] outperforms recent advanced matter-based and all-optical-based schemes.

\section{PRACTICAL IMPLEMENTATION}

In this section, we describe the methods to implement our schemes from a practical point of view. We briefly review how each physical-level element can be implemented, and we roughly estimate the effects of imperfect physical-level elements on the performance of the CBSM scheme. Throughout this section, a logical-level gate or measurement means a gate or measurement in the modified parity encoding basis $\left\{\left|0_{L}\right\rangle,\left|1_{L}\right\rangle\right\}$, whereas a physical-level one means a gate or measurement in the coherent-state basis $\{| \pm \alpha\rangle\}$.

\section{A. Encoding logical qubits}

\section{Logical level}

The encoding circuit of a logical qubit is illustrated in Fig. 11 for the $n=m=3$ case. The desired qubit encoded in the coherent-state basis is prepared at the first PLS of the first block. First, a physical-level controlled-NOT (CNOT) gate is operated between the first PLS of the first block (control) and the first PLS of each of the other blocks (target). After that, for each block, CNOT gates are operated between the first 

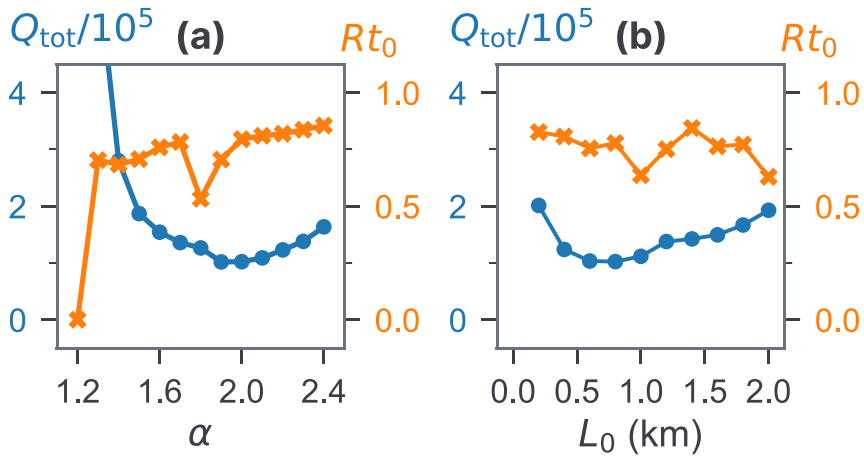

$$
\longrightarrow Q_{\text {tot }} \cdots R t_{0}
$$

FIG. 10. Optimal effective total cost $Q_{\text {tot }}$ and the corresponding expected key length $R t_{0}$ against (a) the coherent-state amplitude $\alpha$ and (b) the repeater station interval $L_{0}$ for the total distance $L=$ $1000 \mathrm{~km}$. For each point, other parameters $\left[n, m, j\right.$, and $L_{0}$ for (a), and $n, m, j$, and $\alpha$ for (b)] are selected to minimize $Q_{\text {tot }}$, and the value of $R t_{0}$ corresponds to that optimal set of parameters. Overall, $Q_{\mathrm{tot}}$ is minimum at $\alpha=1.9$ and $L_{0}=0.7 \mathrm{~km}$.

PLS (target) and the other ones (control). The encoding circuit for arbitrary values of $n$ and $m$ generally requires $n-1$ copies of the coherent state $|\alpha\rangle, n(m-1)$ copies of the even SCS $N_{+}(|\alpha\rangle+|-\alpha\rangle)$, and $n m-1$ physical-level CNOT gates. The decoding circuit is exactly the reverse of the encoding circuit.

\section{Effects of imperfect physical-level elements}

Here we roughly estimate how imperfect implementation of the SCSs and CNOT gates used in the encoding circuit affects the performance of the CBSM scheme. The effects

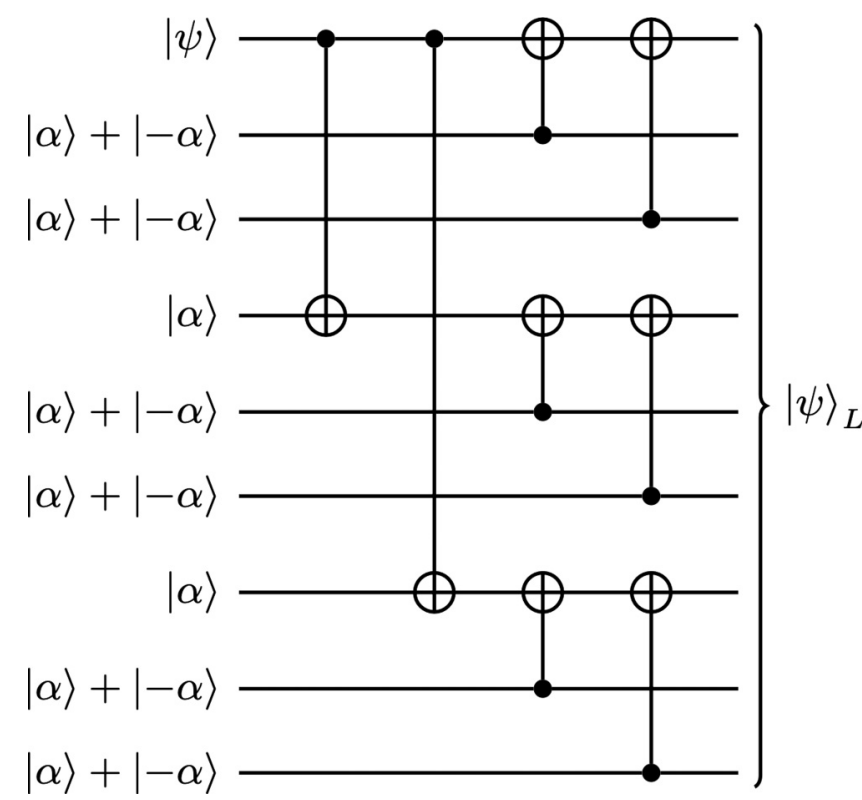

FIG. 11. Encoding circuit of the modified parity code defined in Definition 1 for the $n=m=3$ case. Here, $|\psi\rangle$ is the desired qubit encoded in the coherent-state basis $\{|\alpha\rangle,|-\alpha\rangle\}$, and all the controlled-NOT (CNOT) gates are also operated in the coherent-state basis. Each state $|\alpha\rangle+|-\alpha\rangle$ is the even SCS, where the normalization constant is omitted.

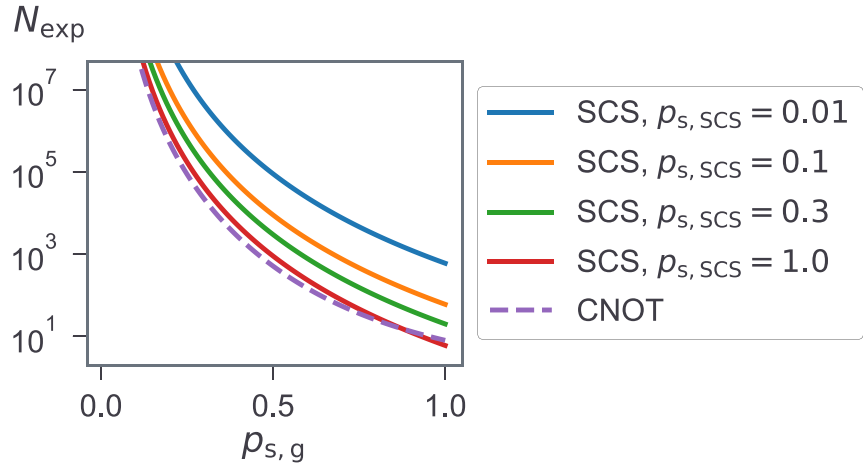

FIG. 12. Expected numbers $\left(N_{\text {exp }}\right)$ of trials of creating an SCS or implementing a CNOT gate to encode a logical qubit of $(n, m)=$ $(3,3)$ for different success probabilities $\left(p_{\mathrm{s}, \mathrm{SCS}}\right.$ and $\left.p_{\mathrm{s}, \mathrm{g}}\right)$ of the schemes to generate each SCS or implement each CNOT gate.

can be viewed from two perspectives: nondeterminacy and inaccuracy.

First, nondeterministic implementation of SCSs or CNOT gates makes the encoding nondeterministic. Let us suppose that each SCS (CNOT gate) is created nondeterministically with a success probability of $p_{\mathrm{s}, \mathrm{SCS}}\left(p_{\mathrm{s}, \mathrm{g}}\right)$. Then the expected numbers $\left(N_{\text {exp,SCS }}\right.$ and $\left.N_{\text {exp,g }}\right)$ of trials of creating an SCS or implementing a CNOT gate to encode a logical qubit are given by

$$
\begin{aligned}
N_{\mathrm{exp}, \mathrm{SCS}} & =\frac{m-1}{p_{\mathrm{s}, \mathrm{SCS}}}\left[\frac{1-p_{\mathrm{s}, \mathrm{g}}^{n}}{p_{\mathrm{s}, \mathrm{g}}^{n m-1}\left(1-p_{\mathrm{s}, \mathrm{g}}\right)}\right], \\
N_{\mathrm{exp}, \mathrm{g}} & =\frac{1-p_{\mathrm{s}, \mathrm{g}}^{n m-1}}{p_{\mathrm{s}, \mathrm{g}}^{n m-1}\left(1-p_{\mathrm{s}, \mathrm{g}}\right)},
\end{aligned}
$$

which are derived in Appendix E. $N_{\text {exp,SCS }}$ and $N_{\text {exp,g }}$ are plotted in Fig. 12 for $(n, m)=(3,3)$, which shows that they are much more sensitive to $p_{\mathrm{s}, \mathrm{g}}$ than $p_{\mathrm{s}, \mathrm{SCS}}$.

Next, inaccurate SCSs or CNOT gates may make the CBSM more vulnerable to failures and errors. Let us suppose that each even SCS for the encoding circuit in Fig. 11 is inaccurately prepared so that its fidelity with an ideal state is lower-bounded by $F_{\mathrm{SCS}}$; namely, for each inaccurate even SCS $\rho_{\text {SCS }}$,

$$
F\left(\left|+_{\alpha}\right\rangle\left\langle+_{\alpha}\right|, \rho_{\mathrm{SCS}}\right)=\left\langle+_{\alpha}\left|\rho_{\mathrm{SCS}}\right|+_{\alpha}\right\rangle \geqslant F_{\mathrm{SCS}}
$$

holds, where $F$ is the fidelity function defined by $F(\rho, \sigma):=$ $\left\{\operatorname{Tr}\left[\left(\rho^{1 / 2} \sigma \rho^{1 / 2}\right)^{1 / 2}\right]\right\}^{2}$ for density operators $\rho, \sigma$, and $\left|+_{\alpha}\right\rangle:=$ $N_{+}(|\alpha\rangle+|-\alpha\rangle)$ is the ideal even SCS with a normalization factor $N_{+}$. We also suppose that each CNOT gate is inaccurately implemented such that its minimum gate fidelity is lowerbounded by $F_{\mathrm{g}}$; namely, for each inaccurate CNOT gate written as a quantum map $\mathcal{E}_{g}$,

$$
\min _{\rho} F\left(\mathcal{E}_{\mathrm{g}, \text { ideal }}(\rho), \mathcal{E}_{\mathrm{g}}(\rho)\right) \geqslant F_{\mathrm{g}}
$$

holds, where the minimization is taken over all states $\rho$, and $\mathcal{E}_{\text {g,ideal }}$ is the quantum map corresponding to the ideal CNOT gate. The Bures distance [64] between two states $\rho_{1}$ and $\rho_{2}$ is defined by

$$
d_{B}\left(\rho_{1}, \rho_{2}\right):=\sqrt{2\left[1-\sqrt{F\left(\rho_{1}, \rho_{2}\right)}\right]} .
$$



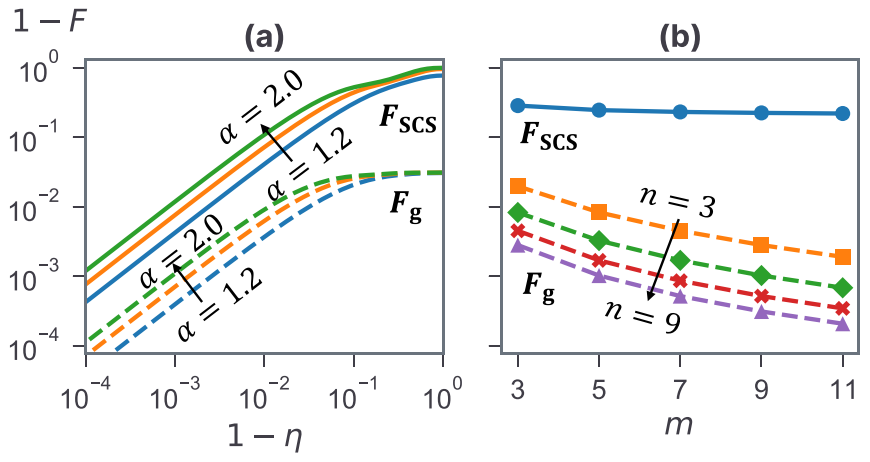

FIG. 13. (a) Photon survival rate $\eta$ where the corresponding photon loss channel on average makes the fidelity loss on an arbitrary perfectly prepared encoded state to the same extent as the fidelity loss by inaccurate SCSs or CNOT gates in the encoding circuit. We assume that the SCSs (CNOT gates) have the fidelity of $F_{\mathrm{SCS}}\left(F_{\mathrm{g}}\right)$ with the ideal one while the CNOT gates (SCSs) are perfect. $\alpha$ is varied within $\{1.2,1.6,2.0\}$, while the encoding size is fixed by $(n, m)=(3,3)$. (b) $F_{\mathrm{SCS}}$ and $F_{\mathrm{g}}$ corresponding to $\eta=0.95$ for different encoding sizes, i.e., different values of $n \in\{3,5,7,9\}$ and $m$, while fixing $\alpha=1.6 . F_{\mathrm{SCS}}$ is nearly independent of $n$.

From the triangular inequality of this distance function and Eqs. (20) and (21), we get a lower bound of the fidelity $F_{\text {enc }}$ of the encoded state generated by the inaccurate circuit with the ideal one:

$$
\begin{aligned}
\sqrt{F_{\mathrm{enc}}} & \geqslant 1-\left[\sqrt{1-F_{\mathrm{SCS}}^{\frac{n(m-1)}{2}}}+(n m-1) \sqrt{1-\sqrt{F_{\mathrm{g}}}}\right]^{2} \\
& :=\sqrt{F_{\mathrm{enc}}^{\mathrm{lb}}} .
\end{aligned}
$$

Next, we see how much such inaccurate preparation of the encoded states decreases the performance of the CBSM by finding the photon survival rate $\eta$ corresponding to $F_{\mathrm{enc}}^{\mathrm{lb}}$. In other words, we find $\eta$ with the corresponding photon loss channel $\Lambda_{\eta}$ satisfying

$$
\begin{aligned}
F_{\mathrm{enc}}^{\mathrm{lb}} & =\overline{F\left(\Lambda_{\eta}\left(\left|\psi_{L}\right\rangle\left\langle\psi_{L}\right|\right),\left|\psi_{L}\right\rangle\left\langle\psi_{L}\right|\right)} \\
& =\overline{\left\langle\psi_{L}\left|\Lambda_{\eta}\left(\left|\psi_{L}\right\rangle\left\langle\psi_{L}\right|\right)\right| \psi_{L}\right\rangle}:=F_{\eta}^{\mathrm{avg}},
\end{aligned}
$$

where the averaging is taken on the Bloch sphere as Eq. (16). We can then estimate the effect of the fidelity loss by that of the corresponding photon loss channel. Although photon loss cannot represent arbitrary types of inaccuracy, at least we can get a rough tendency on how the performance of the scheme depends on $F_{\text {enc }}$.

In Appendix F, we derive a closed-form expression of $F_{\eta}^{\text {avg }}$ in terms of $\alpha, \eta, R, n$, and $m$. Combining Eqs. (22), (23), and (F1), we get the values of $\eta$ corresponding to given values of $F_{\mathrm{SCS}}$ and $F_{\mathrm{g}}$. Figure 13 illustrates such correspondences for different parameter sets, where either $F_{\mathrm{SCS}}$ or $F_{\mathrm{g}}$ is fixed to be unity. It shows that our scheme is quite tolerant of the inaccuracy of SCSs. For example, if $\alpha=1.6, F_{\mathrm{SCS}}$ near 0.7 corresponds to $\eta=0.95$, which does not damage the performance critically, as shown in Figs. 5(a) and 7. However, our scheme is sensitive to the inaccuracy of CNOT gates; e.g., $F_{g}$ should be larger than about 0.97 even for the smallest encoding $(n, m)=(3,3)$ to make the protocol at least work.

\section{Implementation of superpositions of coherent states}

It was known that SCSs (often called Schrödinger's cat states) in free-propagating optical fields may be produced using a strong nonlinearity [65] or a precise photon-resolving detector [66,67], although it was experimentally highly demanding. Later, the possibilities of generating SCSs using realistic detectors [68,69], a weak nonlinearity [70-72], or photon number states [73] were explored. An SCS with $\alpha \approx$ 1.6 was experimentally generated using a photon number state and homodyne detection [73], although its fidelity was limited. Recently, photon subtraction was widely used to generate SCSs due to its simplicity in the implementation [74-79]. Although only odd SCSs with small amplitudes can be generated reliably by photon subtraction, they can be converted to even SCSs with larger amplitudes via the nondeterministic enlargement technique $[68,78,80]$ without significant fidelity loss [78].

Thanks to these works, free-propagating SCSs with amplitudes of $|\alpha| \lesssim 2$ are now within reach of current technology, while their purities and the success probabilities of the schemes have yet to be improved. As a recent example, the authors in Ref. [78] generated an even SCS of $\alpha=1.85$ with a fidelity of $\sim 0.59$ and a success probability of $\sim 0.2$ via photon subtraction and the enlargement technique. The authors in Ref. [77] generated an odd SCS of $\alpha=1.01$ with a higher fidelity $(\sim 0.78)$ via photon subtraction, although they did not demonstrate its enlargement. As discussed in Sec. VII A 2, we estimate that this value of fidelity is barely acceptable to take advantage of the CBSM scheme. Furthermore, pure odd SCSs can be generated in principle with photon subtraction [77], thus it may be possible shortly to generate even SCSs with high enough fidelities for faithful CBSM.

\section{Implementation of the physical-level CNOT gates}

Implementing the physical-level CNOT gates is a challenging task. A CNOT gate for a coherent-state qubit can be implemented near-deterministically via gate teleportation with prearranged entangled states $[17,18]$. An alternative way proposed in Ref. [81] employs single-photon subtraction as the driving force to implement the physical-level controlledphase (CPHASE) gates, which is relatively easier to implement and can achieve arbitrary precision but is nondeterministic. To convert the generated CPHASE gates to the CNOT gates, the physical-level Hadamard gates are required. It is worth noting that a nondeterministic physical-level Hadamard gate was experimentally demonstrated for small values of $\alpha(\leqslant 0.75)$ with a gate fidelity of $F \approx 0.7$ [82]. In short, physical-level CNOT gates with a sufficiently high fidelity for the CBSM scheme are possible in principle, while their experimental implementations require further investigation.

\section{B. Photon-number parity detectors}

Once logical qubits are prepared off-line, PNPDs and 50:50 beam splitters are only in-line physical-level elements required for the CBSM, as shown in Fig. 3. Unlike beam splitters, PNPDs are quite challenging to implement. We thus define the cost function of a CBSM in Definition 2 as half the number of PNPDs used for it. 


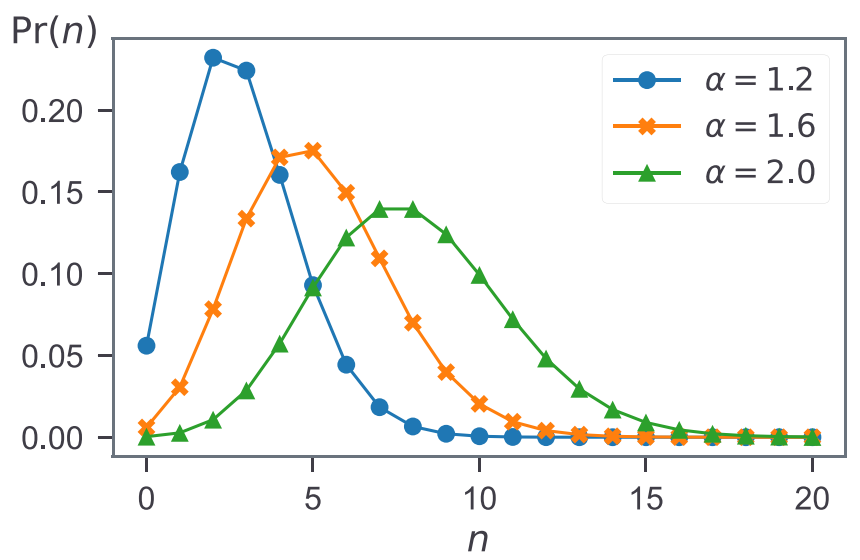

FIG. 14. Probability mass function $\operatorname{Pr}(n)$ of $n:=\max \left(n_{1}, n_{2}\right)$, where $n_{1}$ and $n_{2}$ are the numbers of photons entering the two PNPDs, respectively, in a physical-level BSM for different values of $\alpha$, assuming that the state is an equal mixture of the four Bell states just before the measurement.

There are two ways to realize PNPDs: detecting the parity of the photon number directly (i.e., direct measurement) or detecting it indirectly by measuring the photon number (i.e., indirect measurement).

Regarding the direct measurement, parity measurements in cavities have been demonstrated and realized via Rydberg atoms interacting with photons [83], Ramsey interferometry $[40,84]$, or strong nonlinear Hamiltonian of a Josephson circuit [37]. However, parity measurements of propagating waves have not been covered much yet except a few studies such as parity measurement via strong nonlinear optical switching devices $[85,86]$ or a cavity QED system realized in superconducting circuits [87].

Indirect measurement, or photon-number-resolving (PNR) detection, is a more actively studied topic due to its wide usability [88]. PNR schemes again can be classified into two categories: inherent PNR detectors and multiplexed single-photon detectors. Transition edge sensors (TESs) are promising candidates for inherent PNR detectors [89-93], which can distinguish up to 12 photons with an estimated detection efficiency of 0.98 [94]. While inherent PNR detectors generally demand tricky conditions [95], multiplexed single-photon detectors exploit several inexpensive singlephoton detectors [96-101]. However, it is currently difficult to achieve sufficiently high efficiency with multiplexed singlephoton detectors; e.g., one cannot resolve more than three photons with better-than-guessing quality by using ideal click detectors with an eight-segment detector [88].

Practically, PNPDs, which can distinguish up to a particular number of photons, are sufficient. For example, for faithful physical-level BSM, PNPDs should distinguish up to at least about 8 photons for $\alpha=1.2,12$ photons for $\alpha=1.6$, and 16 photons for $\alpha=2$, as shown in Fig. 14, which is partially feasible in current technology. Additionally, neglecting dark count errors (where the PNPDs detect photons although no photons are entered), errors in the PNPD results may make sign flip errors ( $Z$-errors) as shown in Table I, thus they can be corrected in block-level BSMs as discussed in Sec. IV B. PNPDs with an error rate of $p$ make an additional logical
Z-error rate of roughly

$$
\Delta p_{z} \approx 1-\left[1-\left(\begin{array}{c}
m \\
\frac{m+1}{2}
\end{array}\right) p^{\frac{m+1}{2}}\right]^{n} \approx n\left(\begin{array}{c}
m \\
\frac{m+1}{2}
\end{array}\right) p^{\frac{m+1}{2}},
$$

where the second approximation holds if $p \ll$ $\left(\begin{array}{c}m \\ (m+1) / 2\end{array}\right)^{2 /(m+1)}$. For example, if $p=5 \%, \Delta p_{z} \approx 2.2 \%$ for $(n, m)=(3,3)$ and $\Delta p_{z} \approx 0.15 \%$ for $(n, m)=(7,7)$.

\section{Logical-level $X$ and $Z$ gates}

Logical-level $X$ and $Z$ gates $\left(X_{L}\right.$ and $\left.Z_{L}\right)$ are used in the quantum repeater scheme discussed in Sec. VIB to recover the original quantum information from the transmitted state based on the classical information on the CBSM results at the end of the network. Note that they are not necessary for the CBSM scheme itself.

An $X_{L}\left(Z_{L}\right)$ gate can be decomposed into $n(m)$ physicallevel $X(Z)$ gates:

$$
\begin{aligned}
& X_{L}=\prod_{i=1}^{n} X_{i k} \quad \text { for any } k \leqslant n, \\
& Z_{L}=\prod_{k=1}^{m} Z_{i k} \quad \text { for any } i \leqslant m,
\end{aligned}
$$

where $X_{i k}\left(Z_{i k}\right)$ is a physical-level $X(Z)$ gate on the $k$ th PLS of the $i$ th block.

Implementation of the physical-level $X$ gate is straightforward: $X=\exp \left(i \pi a^{\dagger} a\right)$, where $a$ is the annihilation operator, is just swifting the electromagnetic wave's phase by $\pi$ [18]. Implementation of the physical-level $Z$ gate is more complicated due to its nonunitarity. An approximate $Z$ gate can be achieved via a nonlinear medium [17], gate teleportation with resources of SCSs [18,21], or single-photon subtraction [81]. The single-photon subtraction method was experimentally demonstrated in Ref. [102].

\section{CONCLUSION}

Bell-state measurement (BSM) is an essential element for optical quantum information processing, particularly for long-range communication through a quantum repeater. The original coherent-state encoding with basis $\{| \pm \alpha\rangle\}$ enables one to perform nearly deterministic BSM, but it is vulnerable to dephasing by photon loss, especially for large values of the amplitude $\alpha$ of the coherent states required to reduce its nonorthogonality. Fault-tolerant operations with encoded coherent-state qubits have been studied mainly with cavity systems, which cannot be directly applied to free-propagating fields.

In this paper, we have explored the possibility to use such encoded coherent-state qubits for long-range quantum communication by designing an appropriate encoding scheme and the corresponding fault-tolerant BSM schemes. We have presented the modified parity encoding, which is a natural extension of the original coherent-state encoding, and we also suggested a hardware-efficient concatenated Bell-state measurement (CBSM) scheme performed in a completely or partially distributed manner. We have argued and numerically verified that the CBSM scheme successfully suppresses both failures and dephasing simultaneously. We have also shown 
that SCSs with reasonable values of the amplitude such as $\alpha \lesssim 2$ are enough to achieve the success probability close to unity. It is worth noting this point since it is difficult to generate superpositions of coherent states (SCSs) with large amplitudes. It is known that free-propagating SCSs with $\alpha \lesssim$ 2 can be generated using current technology [73,75,78]. Furthermore, we have shown that the quantum repeater scheme using the CBSM scheme for error correction enables efficient long-range quantum communication over $1000 \mathrm{~km}$, where the performance against the cost is on a similar level with the CBSM scheme of the multiphoton polarization encoding [11].

In summary, we have demonstrated that the properly encoded coherent-state qubits in free-propagating fields provide an alternative way for fault-tolerant information processing enabling long-range communication. In addition to presenting the possibility, we have shown that the performance of our CBSM and repeater scheme is comparable to that of other methods, and even exceeds them in some cases.

Our encoding and CBSM schemes are relatively simple: The modified parity encoding is a simple generalized Shor's nine-qubit code, and the CBSM is also just a classical information processing with the results of well-known physical-level BSMs. We have further shown that the methods to encode logical qubits and implement logical gates and measurements are the elementary compositions of physical-level gates or measurements in the coherent-state basis. Therefore, the most challenging part to realize our scheme is on the physical level, such as generating free-propagating SCSs and implementing elementary logical gates and photon-number parity detectors (PNPDs) used for physical-level BSMs. Fortunately, many appropriate implementation methods have been proposed for all of them, even though some of them are nondeterministic or costly. Of course, experimental inaccuracy during these physical-level processes would also affect the performance of our scheme, which was approximately estimated in Sec. VII. The details of such effects require further investigations in the future.

\section{ACKNOWLEDGMENTS}

This work was supported by National Research Foundation of Korea grants funded by the Korean government
(NRF-2020R1A2C1008609, NRF-2019M3E4A1080074, and NRF-2020K2A9A1A06102946) via the Institute for Applied Physics at Seoul National University and by Institute of Information \& communications Technology Planning \& Evaluation (IITP) grants funded by the Korea government (MSIT) (IITP-2021-2020-0-01606 and IITP-2021-0-01059). S.W.L. acknowledges support from the National Research Foundation of Korea (2020M3E4A1079939) and the KIST institutional program (2E31021).

\section{APPENDIX A: POSITIVE-OPERATOR-VALUED MEASURE ELEMENTS OF BELL-STATE MEASUREMENT ON COHERENT-STATE QUBITS IN A LOSSY ENVIRONMENT}

Here, we explicitly present the positive-operator-valued measure (POVM) elements of BSM under the basis of $\{| \pm \alpha\rangle\}$ in a lossy environment, which is addressed in Sec. II. The set of operators $\left\{M_{x, y} \mid x, y \in\{0,1,2\}\right\}$ where

$$
M_{x, y}:=\left[\mathcal{U}_{\mathrm{BS}} \circ\left(\Lambda_{\eta_{1}} \otimes \Lambda_{\eta_{2}}\right)\right]^{\dagger}\left(\Pi_{x} \otimes \Pi_{y}\right)
$$

forms a POVM corresponding to the BSM of coherent-state qubits, where $\mathcal{U}_{\mathrm{BS}}$ is a unitary channel corresponding to a 50:50 beam splitter, $\Lambda_{\eta}$ is a photon loss channel with a survival rate of $\eta$, and $\Pi_{x}$ is a projector defined by

$$
\begin{aligned}
& \Pi_{0}:=\left|0_{\mathrm{F}}\right\rangle\left\langle 0_{\mathrm{F}}\left|, \quad \Pi_{1}:=\sum_{n \geqslant 1: \text { odd }}\right| n_{\mathrm{F}}\right\rangle\left\langle n_{\mathrm{F}}\right|, \\
& \Pi_{2}:=\sum_{n \geqslant 2 \text { :even }}\left|n_{\mathrm{F}}\right\rangle\left\langle n_{\mathrm{F}}\right|,
\end{aligned}
$$

where $\left|n_{\mathrm{F}}\right\rangle$ is the Fock state with a photon number of $n$. The photon loss channel $\Lambda_{\eta}$ transforms $|\alpha\rangle\langle\alpha|$ and $|\alpha\rangle\langle-\alpha|$ as follows:

$$
\begin{aligned}
\Lambda_{\eta}(|\alpha\rangle\langle\alpha) \mid & =|\sqrt{\eta} \alpha\rangle\langle\sqrt{\eta} \alpha|, \\
\Lambda_{\eta}(|\alpha\rangle\langle-\alpha|) & =e^{-2(1-\eta)|\alpha|^{2}}|\sqrt{\eta} \alpha\rangle\langle-\sqrt{\eta} \alpha| .
\end{aligned}
$$

with these relations, we find the analytic expressions of the matrix elements of each POVM element $M_{x, y}$ as

$$
\begin{aligned}
\left\langle\phi_{ \pm}\left|M_{x, y}\right| \phi_{ \pm}\right\rangle & =c_{ \pm}\left[1 \pm(-1)^{x+y} e^{-2\left(2-\eta_{1}-\eta_{2}\right)|\alpha|^{2}}\right] f_{x}\left(\eta_{+}\right) f_{y}\left(\eta_{-}\right), \\
\left\langle\psi_{ \pm}\left|M_{x, y}\right| \psi_{ \pm}\right\rangle & =c_{ \pm}\left[1 \pm(-1)^{x+y} e^{-2\left(2-\eta_{1}-\eta_{2}\right)|\alpha|^{2}}\right] f_{x}\left(\eta_{-}\right) f_{y}\left(\eta_{+}\right), \\
\left\langle\phi_{ \pm}\left|M_{x, y}\right| \psi_{ \pm}\right\rangle & =c_{ \pm}\left[ \pm(-1)^{x+y} e^{-2\left(1-\eta_{1}\right)|\alpha|^{2}}+e^{-2\left(1-\eta_{2}\right)|\alpha|^{2}}\right] f_{x}\left(\sqrt{\eta_{+} \eta_{-}}\right) f_{y}\left(\sqrt{\eta_{+} \eta_{-}}\right), \\
\left\langle\phi_{+}\left|M_{x, y}\right| \phi_{-}\right\rangle & =\left\langle\psi_{+}\left|M_{x, y}\right| \psi_{-}\right\rangle=\left\langle\phi_{ \pm}\left|M_{x, y}\right| \psi_{\mp}\right\rangle=0,
\end{aligned}
$$

where

$$
c_{ \pm}:=\frac{e^{-\left(\eta_{1}+\eta_{2}\right)|\alpha|^{2}}}{1 \pm e^{-4|\alpha|^{2}}}, \quad \eta_{ \pm}:=\frac{\left(\sqrt{\eta_{1}} \pm \sqrt{\eta_{2}}\right)^{2}}{2}, \quad f_{i}(\eta):= \begin{cases}1 & \text { if } i=0, \\ \sinh \left(\eta|\alpha|^{2}\right) & \text { if } i=1, \\ \cosh \left(\eta|\alpha|^{2}\right)-1 & \text { if } i=2 .\end{cases}
$$

\section{APPENDIX B: DERIVATION OF THE PROBABILITY DISTRIBUTIONS OF CONCATENATED BELL-STATE MEASUREMENT RESULTS}

In this Appendix, we show a brief outline to induce the analytic expressions of the probability distributions of
CBSM results conditioning to the initial Bell states before the measurement. We only consider the unoptimized CBSM scheme since the measurement results of the hardwareefficient CBSM scheme are the direct consequences of those of the unoptimized scheme. 


\section{Derivation of the probability distributions of block-level results}

We first find the probability distributions of block-level BSM results, conditioning to the initial block-level Bell state. A single $\mathrm{BSM}_{1}$ result can be expressed by two vectors $\mathbf{x}, \mathbf{y} \in$ $\{0,1,2,3\}^{m}$, where their $i$ th elements are the two PNPD results of the $i$ th PLS. We want to find $\operatorname{Pr}\left(\mathbf{x}, \mathbf{y} \mid B_{1}\right)$ for $\left|B_{1}\right\rangle \in$ $\mathcal{B}_{1}:=\left\{\left|\phi_{ \pm}^{(m)}\right\rangle,\left|\psi_{ \pm}^{(m)}\right\rangle\right\}$

From Eqs. (5) and (12a), the conditional probability for the initial state of $\left|B_{1}\right\rangle=\left|\phi_{ \pm}^{(m)}\right\rangle$ is expressed as

$$
\begin{aligned}
\operatorname{Pr}\left(\mathbf{x}, \mathbf{y} \mid \phi_{ \pm}^{(m)}\right) & =\left\langle\phi_{ \pm}^{(m)}\left|\bigotimes_{i=1}^{m} M_{x_{i}, y_{i}}\right| \phi_{ \pm}^{(m)}\right\rangle \\
& =\frac{1}{2} \tilde{N}_{ \pm}(1, m)^{2} \sum_{l, l^{\prime}=\text { even } \leqslant m} g_{m, l, l^{\prime}}^{ \pm}(\mathbf{x}, \mathbf{y}),
\end{aligned}
$$

where $\tilde{N}_{ \pm}(1, m)$ is defined in Eq. (9). The function $g_{m, l, l^{\prime}}^{ \pm}(\mathbf{x}, \mathbf{y})$ is defined as

$$
g_{m, l, l^{\prime}}^{ \pm}(\mathbf{x}, \mathbf{y}):=\sum_{\substack{\bigotimes_{i=1}^{m}\left|P_{i}\right\rangle \in P_{ \pm}(m, l) \\ \bigotimes_{i=1}^{m} \in\left|P_{i}^{\prime}\right\rangle \in P_{ \pm}\left(m, l^{\prime}\right)}}\left[\prod_{i=1}^{m}\left\langle P_{i}\left|M_{x_{i}, y_{i}}\right| P_{i}^{\prime}\right\rangle\right],
$$

where

$$
P_{ \pm}(m, l):=\operatorname{Perm}\left[\left|\psi_{ \pm}\right\rangle^{\otimes l}\left|\phi_{ \pm}\right\rangle^{\otimes m-l}\right]
$$

and Perm[-] is the set of all the permutations of tensor products inside the square brackets. The function $g_{m, l, l^{\prime}}^{ \pm}$has a recurrence relation (omit $\mathbf{x}$ and $\mathbf{y}$ for simplicity):

$$
\begin{aligned}
g_{m, l, l^{\prime}}^{ \pm}= & g_{m-1, l, l^{\prime}}^{ \pm} M_{11}^{(m) \pm} \\
& +\left[g_{m-1, l, l^{\prime}-1}^{ \pm}+g_{m-1, l-1, l^{\prime}}^{ \pm}\right] M_{12}^{(m) \pm} \\
& +g_{m-1, l-1, l^{\prime}-1}^{ \pm} M_{22}^{(m) \pm},
\end{aligned}
$$

where

$$
\begin{aligned}
& M_{11}^{(k) \pm}:=\left\langle\phi_{ \pm}\left|M_{x_{k}, y_{k}}\right| \phi_{ \pm}\right\rangle, \\
& M_{12}^{(k) \pm}:=\left\langle\phi_{ \pm}\left|M_{x_{k}, y_{k}}\right| \psi_{ \pm}\right\rangle, \\
& M_{22}^{(k) \pm}:=\left\langle\psi_{ \pm}\left|M_{x_{k}, y_{k}}\right| \psi_{ \pm}\right\rangle,
\end{aligned}
$$

which can be calculated from Eqs. (A2). Now, we define a vector $\mathbf{v}_{m}^{ \pm}(\mathbf{x}, \mathbf{y})$ (note that $g_{m, l, l^{\prime}}^{ \pm}$is a function of $\mathbf{x}$ and $\mathbf{y}$ ):

$$
\begin{gathered}
\mathbf{v}_{m}^{ \pm}:=\left(\sum_{l, l^{\prime} \text { :even } \leqslant m} g_{m, l, l^{\prime}}^{ \pm}, \sum_{\substack{l: \text { even } \leqslant m \\
l^{\prime}: \text { odd } \leqslant m}} g_{m, l, l^{\prime}}^{ \pm},\right. \\
\left.\sum_{\substack{l: \text { odd } \leqslant m \\
l^{\prime} \text { :even } \leqslant m}} g_{m, l, l^{\prime}}^{ \pm}, \sum_{l, l^{\prime} \text { :odd } \leqslant m} g_{m, l, l^{\prime}}^{ \pm}\right)^{T} .
\end{gathered}
$$

From Eq. (B2), we get a recurrence relation of $\mathbf{v}_{m}^{ \pm}$:

$$
\begin{aligned}
\mathbf{v}_{m}^{ \pm} & =\left(\begin{array}{llll}
M_{11}^{(m) \pm} & M_{12}^{(m) \pm} & M_{12}^{(m) \pm} & M_{22}^{(m) \pm} \\
M_{12}^{(m) \pm} & M_{11}^{(m) \pm} & M_{22}^{(m) \pm} & M_{12}^{(m) \pm} \\
M_{12}^{(m) \pm} & M_{22}^{(m) \pm} & M_{11}^{(m) \pm} & M_{12}^{(m) \pm} \\
M_{22}^{(m) \pm} & M_{12}^{(m) \pm} & M_{12}^{(m) \pm} & M_{11}^{(m) \pm}
\end{array}\right) \mathbf{v}_{m-1}^{ \pm} \\
& :=\tilde{\mathbf{M}}_{x_{m}, y_{m}}^{ \pm} \mathbf{v}_{m-1}^{ \pm} .
\end{aligned}
$$

Considering the initial condition at $m=1, \mathbf{v}_{m}^{ \pm}(\mathbf{x}, \mathbf{y})$ is written as

$$
\mathbf{v}_{m}^{ \pm}(\mathbf{x}, \mathbf{y})=\tilde{\mathbf{M}}_{x_{m}, y_{m}}^{ \pm} \cdots \tilde{\mathbf{M}}_{x_{1}, y_{1}}^{ \pm}\left(\begin{array}{llll}
1, & 0,0,0
\end{array}\right)^{T} .
$$

Finally, $\operatorname{Pr}\left(\mathbf{x}, \mathbf{y} \mid \phi_{ \pm}^{(m)}\right)$ is written in terms of the vector $\mathbf{v}_{m}^{ \pm}$ using Eqs. (B1) and (B4):

$$
\operatorname{Pr}\left(\mathbf{x}, \mathbf{y} \mid \phi_{ \pm}^{(m)}\right)=\frac{1}{2} \tilde{N}_{ \pm}(1, m)^{2} v_{m 1}^{ \pm}(\mathbf{x}, \mathbf{y}),
$$

where $v_{m i}^{ \pm}$is the $i$ th element of $\mathbf{v}_{m}^{ \pm}$. In the similar way, $\operatorname{Pr}\left(\mathbf{x}, \mathbf{y} \mid \psi_{ \pm}^{(m)}\right)$ is written as

$$
\operatorname{Pr}\left(\mathbf{x}, \mathbf{y} \mid \psi_{ \pm}^{(m)}\right)=\frac{1}{2} \tilde{N}_{ \pm}(1, m)^{2} v_{m 4}^{ \pm}(\mathbf{x}, \mathbf{y}) .
$$

In conclusion, the conditional probability distribution of CBSM results conditioning to the input block-level Bell state is obtained from Eqs. (B7) with Eqs. (A2), (B3), (B5), and (B6), all of which are written in simple matrix forms.

\section{Derivation of the probability distributions of logical-level results}

Now, we consider the probability distributions of logicallevel results conditioning to the initial logical-level Bell state, which is the goal of this Appendix. A single CBSM result can be expressed by two matrices $\mathbf{X}, \mathbf{Y} \in\{0,1,2,3\}^{n \times m}$, where their $(i, k)$ elements are the two PNPD results of the $k$ th PLS of the $i$ th block. What we want to find is the probability distribution $\operatorname{Pr}\left(\mathbf{X}, \mathbf{Y} \mid B_{2}\right)$ for $\left|B_{2}\right\rangle \in \mathcal{B}_{2}:=\left\{\left|\Phi_{ \pm}\right\rangle,\left|\Psi_{ \pm}\right\rangle\right\}$.

Because of the similarity of Eqs. (8) and (12), we can follow almost the same logical structure as the previous subsection when finding the expressions of the probability distributions. However, there exist three main differences between the block and logical level. First, the roles of the letters and signs are inverted between the two sets of equations. Second, there are unnormalized states in the summations of Eqs. (8), unlike Eqs. (12). Lastly, $\left\langle\phi_{+}^{(m)}\left(\psi_{+}^{(m)}\right)\left|\bigotimes_{k=1}^{m} M_{x_{k}, y_{k}}\right| \phi_{-}^{(m)}\left(\psi_{-}^{(m)}\right)\right\rangle$ vanishes unlike the corresponding one in the block level, i.e., $\left\langle\phi_{ \pm}\left|M_{x, y}\right| \psi_{ \pm}\right\rangle$in Eq. (B3b).

Considering the differences, we define $2 \times 2$ matrices $\tilde{\mathbf{L}}_{\mathbf{x}, \mathbf{y}}^{\phi}$ and $\tilde{\mathbf{L}}_{\mathbf{x}, \mathbf{y}}^{\psi}$ where $\mathbf{x}, \mathbf{y} \in\{0,1,2,3\}^{m}$, instead of $4 \times 4$ matrices, in the similar way to the block-level case:

$$
\tilde{\mathbf{L}}_{\mathbf{x}, \mathbf{y}}^{\phi(\psi)}:=\left(\begin{array}{ll}
L_{+}^{\phi(\psi)} & L_{-}^{\phi(\psi)} \\
L_{-}^{\phi(\psi)} & L_{+}^{\phi(\psi)}
\end{array}\right),
$$

where

$$
\begin{aligned}
L_{ \pm}^{\phi(\psi)}:= & {\left[1 \pm u(\alpha, m)^{2}\right] } \\
& \times\left\langle\phi_{ \pm}^{(m)}\left(\psi_{ \pm}^{(m)}\right)\left|\bigotimes_{k=1}^{m} M_{x_{k}, y_{k}}\right| \phi_{ \pm}^{(m)}\left(\psi_{ \pm}^{(m)}\right)\right\rangle,
\end{aligned}
$$

$u(\alpha, m)$ is defined in Eq. (11), and $x_{k}\left(y_{k}\right)$ is the $k$ th element of $\mathbf{x}(\mathbf{y})$. We do not need $4 \times 4$ matrices, since the off-diagonal elements of $\bigotimes_{k=1}^{m} M_{x_{k}, y_{k}}$ between two Bell states of different signs vanish. We also note that the right-hand side of Eq. (B8) can be calculated from Eqs. (B7). The conditional probability $\operatorname{Pr}\left(\mathbf{X}, \mathbf{Y} \mid B_{2}\right)$, where the $i$ th row vector of $\mathbf{X}(\mathbf{Y})$ is $\mathbf{x}_{i}\left(\mathbf{y}_{i}\right)$, is then

$$
\begin{aligned}
& \operatorname{Pr}\left(\mathbf{X}, \mathbf{Y} \mid \Phi_{+}\left(\Psi_{+}\right)\right)=\tilde{N}_{+}(n, m)^{2} w_{n 1}^{\phi(\psi)}(\mathbf{X}, \mathbf{Y}) \\
& \operatorname{Pr}\left(\mathbf{X}, \mathbf{Y} \mid \Phi_{-}\left(\Psi_{-}\right)\right)=\tilde{N}_{-}(n, m)^{2} w_{n 2}^{\phi(\psi)}(\mathbf{X}, \mathbf{Y})
\end{aligned}
$$


where $\tilde{N}_{ \pm}(n, m)$ is defined in Eq. (9), and $w_{n \mu}^{\phi(\psi)}(\mathbf{X}, \mathbf{Y})$ is the $\mu$ th element of the two-dimensional vector $\mathbf{w}_{n}^{\phi(\psi)}(\mathbf{X}, \mathbf{Y})$ defined by

$$
\mathbf{w}_{n}^{\phi(\psi)}(\mathbf{X}, \mathbf{Y}):=\tilde{\mathbf{L}}_{\mathbf{x}_{n}, \mathbf{y}_{n}}^{\phi(\psi)} \cdots \tilde{\mathbf{L}}_{\mathbf{x}_{1}, \mathbf{y}_{1}}^{\phi(\psi)}(1,0)^{T} .
$$

\section{APPENDIX C: METHOD FOR SAMPLING CONCATENATED BELL-STATE MEASUREMENT RESULTS}

In this Appendix, we explain the method to sample CBSM results. Since we have the analytic expressions of the probability distributions of measurement results [Eqs. (B9)], it is possible to sample arbitrary CBSM results, each of which is composed of $2 \mathrm{~nm}$ PNPD results. However, since the number of CBSM results increases exponentially on $n$ and $m$, it is computationally expensive to use this method. Instead of that, denoting $(p, q)$ the $q$ th PLS of the $p$ th block, we sample the results for each PLS in order: $(1,1) \rightarrow(1,2) \rightarrow(1,3) \rightarrow \cdots \rightarrow(1, m) \rightarrow$ $(2,1) \rightarrow \cdots \rightarrow(2, m) \rightarrow \cdots \rightarrow(n, m)$. Therefore, we need the conditional probability of getting each $(p, q)$ result conditioning to all the results before $(p, q)$.

The conditional probability we want is

$$
\begin{aligned}
\operatorname{Pr}\left(x_{p q}, y_{p q} \mid x_{11}, y_{11}, \ldots, x_{p^{\prime} q^{\prime}}, y_{p^{\prime} q^{\prime}} ; B_{2}\right) & \propto \operatorname{Pr}\left(x_{11}, y_{11}, \ldots, x_{p q}, y_{p q} \mid B_{2}\right) \\
& =\operatorname{Pr}\left(\mathbf{x}_{1}, \mathbf{y}_{1}, \ldots, \mathbf{x}_{p-1}, \mathbf{y}_{p-1}, x_{p 1}, y_{p 1}, \ldots, x_{p q}, y_{p q} \mid B_{2}\right),
\end{aligned}
$$

where

$$
\left(p^{\prime}, q^{\prime}\right)= \begin{cases}(p, q-1) & \text { if } q>1, \\ (p-1, m) & \text { otherwise, }\end{cases}
$$

$x_{i k}$ and $y_{i k}$ are the measurement results of the two $(i, k)$ PNPDs, $\left|B_{2}\right\rangle \in \mathcal{B}_{2}$, and $\mathbf{x}_{i}\left(\mathbf{y}_{i}\right)$ is a vector whose $k$ th element is $x_{i k}$ $\left(y_{i k}\right)$. Note that the proportionality is valid only when $x_{11}, y_{11}, \ldots, x_{p^{\prime} q^{\prime}}, y_{p^{\prime} q^{\prime}}$ are fixed. From now on, we use the proportionality notation while assuming this condition. Using Eq. (8a) and the fact from Eq. (A2d) that the cross terms of $\bigotimes_{s=1}^{m} M_{x_{r s}, y_{r s}}$ between Bell states with different signs vanish, it is deduced that the right-hand side of Eq. (C1) with $\left|B_{2}\right\rangle=\left|\Phi_{ \pm}\right\rangle$is

$$
\begin{aligned}
& \operatorname{Pr}\left(\mathbf{x}_{1}, \mathbf{y}_{1}, \ldots, \mathbf{x}_{p-1}, \mathbf{y}_{p-1}, x_{p 1}, y_{p 1}, \ldots, x_{p q}, y_{p q} \mid \Phi_{+(-)}\right) \\
& \propto \sum_{k: \text { even(odd } \leqslant \leqslant n} \sum_{\bigotimes_{r=1}^{n}\left|P_{r}^{(m)}\right\rangle \in \operatorname{Perm}\left[\left|\Phi_{-}\right\rangle^{\otimes k}\left|\Phi_{+}\right\rangle^{\otimes n-k}\right]}\left[C_{+}^{2(n-k)} C_{-}^{2 j} h_{P_{1}, \ldots, P_{p}}^{(p, q)}\right],
\end{aligned}
$$

where $C_{ \pm}:=\left[1 \pm u(\alpha, m)^{2}\right]^{1 / 2}$, the normalization constant in Eq. (10). Also,

$$
h_{P_{1}, \ldots, P_{p}}^{(p, q)}:=\left[\prod_{r=1}^{p-1}\left\langle P_{r}^{(m)}\left|\bigotimes_{s=1}^{m} M_{r s}\right| P_{r}^{(m)}\right\rangle\right]\left\langle P_{p}^{(m)}\left|\left(\bigotimes_{s=1}^{q} M_{p s}\right) \otimes I^{\otimes m-q}\right| P_{p}^{(m)}\right\rangle,
$$

where $M_{r s}:=M_{x_{r s}, y_{r s}}$, and $I$ is the identity operator in a single PLS. Again, using Eq. (12a), the last part of the right-hand side of the above definition is

$$
\begin{gathered}
\left\langle\phi_{ \pm}^{(m)}\left|\left(\bigotimes_{s=1}^{q} M_{p s}\right) \otimes I^{\otimes m-q}\right| \phi_{ \pm}^{(m)}\right\rangle \propto \sum_{\substack { l, l^{\prime}: \text { even } \leqslant m \\
\begin{subarray}{c}{\bigotimes_{s=1}^{m}\left|P_{s}\right\rangle \in \operatorname{Perm}\left[\left|\psi_{ \pm}\right\rangle^{\otimes l}\left|\phi_{ \pm}\right\rangle^{\otimes m-l}\right] \\
\bigotimes_{s=1}^{m}\left|P_{s}^{\prime}\right\rangle \in \operatorname{Perm}\left[\left|\psi_{ \pm}\right\rangle^{\otimes l^{\prime}}\left|\phi_{ \pm}\right\rangle^{\otimes m-l^{\prime}}\right]{ l , l ^ { \prime } : \text { even } \leqslant m \\
\begin{subarray} { c } { \bigotimes _ { s = 1 } ^ { m } | P _ { s } \rangle \in \operatorname { P e r m } [ | \psi _ { \pm } \rangle ^ { \otimes l } | \phi _ { \pm } \rangle ^ { \otimes m - l } ] \\
\bigotimes _ { s = 1 } ^ { m } | P _ { s } ^ { \prime } \rangle \in \operatorname { P e r m } [ | \psi _ { \pm } \rangle ^ { \otimes l ^ { \prime } } | \phi _ { \pm } \rangle ^ { \otimes m - l ^ { \prime } } ] } }\end{subarray}}\left[\prod_{s=1}^{q}\left\langle P_{s}\left|M_{p s}\right| P_{s}^{\prime}\right\rangle \prod_{s=q+1}^{m}\left\langle P_{s} \mid P_{s}^{\prime}\right\rangle\right] \\
:=\xi_{ \pm, p, q}^{\phi} .
\end{gathered}
$$

After transforming the right-hand side of the above equation appropriately with using the fact that $\left\langle\phi_{-} \mid \psi_{-}\right\rangle$vanishes while $\left\langle\phi_{+} \mid \psi_{+}\right\rangle$does not, we obtain

$$
\begin{aligned}
& \xi_{+, p, q}^{\phi}= \begin{cases}R_{q}^{+}\left(v_{q 1}^{+}+v_{q 4}^{+}\right)+R_{q}^{-}\left(v_{q 2}^{+}+v_{q 3}^{+}\right) & \text {if } q<m, \\
v_{m 1}^{+} & \text {if } q=m,\end{cases} \\
& \xi_{-, p, q}^{\phi}= \begin{cases}v_{q 1}^{-}+v_{q 4}^{-} & \text {if } q<m, \\
v_{m 1}^{-} & \text {if } q=m,\end{cases}
\end{aligned}
$$

where $R_{q}^{ \pm}:=\left(1+\left\langle\phi_{+} \mid \psi_{+}\right\rangle\right)^{m-q} \pm\left(1-\left\langle\phi_{+} \mid \psi_{+}\right\rangle\right)^{m-q}$, and $v_{q i}^{ \pm}$is the $i$ th element of vector $\mathbf{v}_{q}^{ \pm}\left(x_{1}, y_{1}, \ldots, x_{p}, y_{p}\right)$ calculated from Eq. (B6). Substituting these in Eq. (C3) and transforming it appropriately, Eqs. (C1) and (C2) become

$$
\begin{aligned}
\operatorname{Pr} & \left.x_{p q}, y_{p q} \mid x_{11}, y_{11}, \ldots, x_{p^{\prime} q^{\prime}}, y_{p^{\prime} q^{\prime}} ; \Phi_{ \pm}\right) \\
& \propto \operatorname{Pr}\left(\mathbf{x}_{1}, \mathbf{y}_{1}, \ldots, \mathbf{x}_{p-1}, \mathbf{y}_{p-1}, x_{p 1}, y_{p 1}, \ldots, x_{p q}, y_{p q} \mid \Phi_{ \pm}\right) \\
& \propto \begin{cases}C_{+}^{2} \xi_{+, p, q}^{\phi}\left(D_{p}^{ \pm} w_{p-1,1}^{\phi}+D_{p}^{\mp} w_{p-1,2}^{\phi}\right)+C_{-}^{2} \xi_{-, p, q}^{\phi}\left(D_{p}^{ \pm} w_{p-1,2}^{\phi}+D_{p}^{\mp} w_{p-1,1}^{\phi}\right) & \text { if } p<n, \\
C_{ \pm}^{2} \xi_{ \pm, p, q}^{\phi} w_{n-1,1}^{\phi}+C_{\mp}^{2} \xi_{\mp, p, q}^{\phi} w_{n-1,2}^{\phi} & \text { if } p=n,\end{cases}
\end{aligned}
$$


where $D_{p}^{ \pm}:=\left(C_{+}^{2}+C_{-}^{2}\right)^{n-p} \pm\left(C_{+}^{2}-C_{-}^{2}\right)^{n-p}$, and $w_{p-1, i}^{\phi}$ is the $i$ th element of vector $\mathbf{w}_{p-1}^{\phi}\left(\mathbf{x}_{1}, \mathbf{y}_{1}, \ldots, \mathbf{x}_{p-1}, \mathbf{y}_{p-1}\right)$ defined in Eqs. (B10).

The probability distribution for the initial state of $\left|B_{2}\right\rangle=\left|\Psi_{ \pm}\right\rangle$is obtained in a very similar way to the above arguments. The result is as follows:

$$
\begin{aligned}
& \operatorname{Pr}\left(x_{p q}, y_{p q} \mid x_{11}, y_{11}, \ldots, x_{p^{\prime} q^{\prime}}, y_{p^{\prime} q^{\prime}} ; \Psi_{ \pm}\right) \\
& \quad \propto \begin{cases}C_{+}^{2} \xi_{+, p, q}^{\psi}\left(D_{p}^{ \pm} w_{p-1,1}^{\psi}+D_{p}^{\mp} w_{p-1,2}^{\psi}\right)+C_{-}^{2} \xi_{-, p, q}^{\psi}\left(D_{p}^{ \pm} w_{p-1,2}^{\psi}+D_{p}^{\mp} w_{p-1,1}^{\psi}\right) & \text { if } p<n, \\
C_{ \pm}^{2} \xi_{ \pm, p, q}^{\psi} w_{n-1,1}^{\psi}+C_{\mp}^{2} \xi_{\mp, p, q}^{\psi} w_{n-1,2}^{\psi} & \text { if } p=n,\end{cases}
\end{aligned}
$$

where $w_{p-1, i}^{\psi}$ is the $i$ th element of vector $\mathbf{w}_{p-1}^{\psi}\left(\mathbf{x}_{1}, \mathbf{y}_{1}, \ldots, \mathbf{x}_{p-1}, \mathbf{y}_{p-1}\right)$ defined in Eq. (B10), and

$$
\begin{aligned}
& \xi_{+, p, q}^{\psi}= \begin{cases}R_{q}^{+}\left(v_{q 1}^{+}+v_{q 4}^{+}\right)+R_{q}^{-}\left(v_{q 2}^{+}+v_{q 3}^{+}\right) & \text {if } q<m, \\
v_{m 4}^{+} & \text {if } q=m,\end{cases} \\
& \xi_{-, p, q}^{\psi}= \begin{cases}v_{q 1}^{-}+v_{q 4}^{-} & \text {if } q<m, \\
v_{m 4}^{-} & \text {if } q=m .\end{cases}
\end{aligned}
$$

In summary, the probability distributions of $(p, q)$ results conditioning to the previous measurement results $(1,1), \ldots,\left(p^{\prime}, q^{\prime}\right)$ and the initial logical-level Bell state can be obtained from Eqs. (C5) and (C6) together with Eqs. (C4) and (C7). We use these probability distributions to sample each physical level one by one in order. There are only nine possible results for each PLS, and the number of PLSs increases linearly on $n$ and $m$. Hence, it is exponentially fast compared to sampling the results with total joint probabilities.

\section{APPENDIX D: AVERAGE TELEPORTATION FIDELITY}

Here, we derive Eq. (17) on the average teleportation fidelity $F_{\text {tel }}$ of CBSM, assuming that the two parties have the same photon survival rate $\left(\eta_{1}=\eta_{2}=\eta\right) . F_{\text {tel }}$ is defined as $F_{\text {tel }}:=\overline{\left\langle\psi_{\text {in }}\left|\rho_{\text {out }}\right| \psi_{\text {in }}\right\rangle}$, where

$$
\left|\psi_{\text {in }}\right\rangle=N\left(\cos \frac{\theta}{2}\left|0_{L}\right\rangle+e^{i \phi} \sin \frac{\theta}{2}\left|1_{L}\right\rangle\right)
$$

with $N:=(1+R \sin \theta \cos \phi)^{-1 / 2}$ is an input state of the standard teleportation protocol using CBSM $\left(R:=\left|0_{L}\right\rangle\left\langle 0_{L}\right| 1_{L}\right)$, $\rho_{\text {out }}$ is the corresponding output state, and the average is taken as

$$
\overline{f\left(\left|\psi_{\text {in }}\right\rangle\right)}:=\frac{1}{4 \pi} \int_{0}^{\pi} d \theta \sin \theta \int_{0}^{2 \pi} d \phi f\left(\left|\psi_{\text {in }}\right\rangle\right) .
$$

From now on, we write $\left|0_{L}\right\rangle,\left|1_{L}\right\rangle$, and $\left|\psi_{\text {in }}\right\rangle$ without the subscripts. We define the unnormalized and normalized Bell states as

$$
\begin{aligned}
\left|\widetilde{B_{0(1)}}\right\rangle & :=|0\rangle|0\rangle \pm|1\rangle|1\rangle, \\
\left|\widetilde{B_{2(3)}}\right\rangle & :=|0\rangle|1\rangle \pm|1\rangle|0\rangle, \\
\left|B_{i}\right\rangle & := \begin{cases}N_{+}\left|\widetilde{B}_{i}\right\rangle & \text { if } i \in\{0,2\}, \\
N_{-}\left|\widetilde{B}_{i}\right\rangle & \text { if } i \in\{1,3\},\end{cases}
\end{aligned}
$$

where $N_{ \pm}:=\left[2\left(1 \pm R^{2}\right)\right]^{-1 / 2}$. Suppose that Alice has $|\psi\rangle_{A}$ and Alice and Charlie share $\left|B_{0}\right\rangle_{A^{\prime} C}$. Then

$$
|\psi\rangle_{A}\left|B_{0}\right\rangle_{A^{\prime} C}=\frac{1}{2} N_{+} \sum_{i}\left[\left|\widetilde{B}_{i}\right\rangle_{A A^{\prime}} \otimes P_{i}|\psi\rangle_{C}\right]:=|T\rangle
$$

holds, where

$$
P_{i}:= \begin{cases}I & \text { if } i=0 \\ Z & \text { if } i=1, \\ X & \text { if } i=2, \\ X Z & \text { if } i=3\end{cases}
$$

Alice performs CBSM on her sides $A A^{\prime}$ and transfers the result classically to Charlie. If the CBSM does not fail and its results is $\left|B_{i}\right\rangle$, Charlie applies $P_{i}$ to his side and finally obtain $\rho_{\text {out }}$.

Let $M_{i}$ for $i \in\{0,1,2,3\}$ and $M_{\mathrm{f}}$ denote the POVM elements of CBSM corresponding to $\left|B_{i}\right\rangle$ and failure, respectively. Also, let $M_{i}^{\prime}$ be an operator satisfying $M_{i}^{\prime \dagger} M_{i}^{\prime}=$ $M_{i} . \rho_{\text {out }}$ can be written as (omitting subscripts $A, A^{\prime}$, and $C$ )

$$
\rho_{\text {out }}=g \operatorname{Tr}_{A A^{\prime}}\left[\sum_{i}\left(M_{i}^{\prime} \otimes P_{i}\right)|T\rangle\langle T|\left(M_{i}^{\prime \dagger} \otimes P_{i}^{\dagger}\right)\right],
$$

where $g:=1 /\left(1-\left\langle T\left|M_{\mathrm{f}} \otimes I\right| T\right\rangle\right)$. We thus get

$$
\begin{aligned}
\left\langle\psi\left|\rho_{\text {out }}\right| \psi\right\rangle & =\operatorname{Tr}\left(\rho_{\text {out }}|\psi\rangle\langle\psi|\right) \\
& =g\left\langle T\left|\left[\sum_{i} M_{i} \otimes P_{i}|\psi\rangle\langle\psi| P_{i}^{\dagger}\right]\right| T\right\rangle \\
& =\frac{N_{+}^{2} g}{4} \sum_{i, j, k}\left\langle\widetilde{B}_{j}\left|M_{i}\right| \widetilde{B}_{k}\right\rangle\left\langle\psi\left|P_{j}^{\dagger} P_{i}\right| \psi\right\rangle\left\langle\psi\left|P_{i}^{\dagger} P_{k}\right| \psi\right\rangle .
\end{aligned}
$$

We first calculate $g$. Remark that a $\mathrm{BSM}_{2}$ fails only if all the $\mathrm{BSM}_{1}$ fail and a $\mathrm{BSM}_{1}$ fails if any $\mathrm{BSM}_{0}$ fails. Thus, $M_{\mathrm{f}}$ is the summation of the tensor products of physical-level POVM elements shown in Appendix A such that each term contains at least $n$ of $M_{0,0}$. Since every matrix element of $M_{0,0}$ is $O\left(e^{-2 \eta|\alpha|^{2}}\right)$ and the number of the terms in $M_{\mathrm{f}}$ containing $n$ of $M_{0,0}$ is $m^{n}$, we get

$$
\forall(i, j), \quad\left\langle B_{i}\left|M_{\mathrm{f}}\right| B_{j}\right\rangle=O\left(\left(m e^{-2 \eta|\alpha|^{2}}\right)^{n}\right) .
$$

Therefore,

$$
g=\frac{1}{1-\left\langle T\left|M_{\mathrm{f}} \otimes I\right| T\right\rangle}=1+O\left(\left(m e^{-2 \eta|\alpha|^{2}}\right)^{n}\right)
$$

holds. 
To calculate $F_{\text {tel }}=\overline{\left\langle\psi\left|\rho_{\text {out }}\right| \psi\right\rangle}$, we first note

$$
\begin{aligned}
\overline{g\left\langle\psi\left|P_{j}^{\dagger} P_{i}\right| \psi\right\rangle\left\langle\psi\left|P_{i}^{\dagger} P_{k}\right| \psi\right\rangle} \\
\quad=\overline{\left\langle\psi\left|P_{j}^{\dagger} P_{i}\right| \psi\right\rangle\left\langle\psi\left|P_{i}^{\dagger} P_{k}\right| \psi\right\rangle}+O\left(\left(m e^{-2 \eta|\alpha|^{2}}\right)^{n}\right) .
\end{aligned}
$$

Considering $N=1+O(R)$, it is straightforward to see

$$
\overline{\left\langle\psi\left|P_{j}^{\dagger} P_{i}\right| \psi\right\rangle\left\langle\psi\left|P_{i}^{\dagger} P_{k}\right| \psi\right\rangle}= \begin{cases}1 & \text { if } i=j=k, \\ \frac{1}{2}+O(R) & \text { if } i \neq j=k, \\ O(R) & \text { otherwise. }\end{cases}
$$

Note that

$$
O\left(\left(m e^{-2 \eta|\alpha|^{2}}\right)^{n}\right) \geqslant O(R)=O\left(\left(m e^{-2|\alpha|^{2}}\right)^{n}\right) .
$$

Using Eqs. (D2)-(D4) and $N_{ \pm}=\frac{1}{\sqrt{2}}+O\left(R^{2}\right)$, we finally obtain Eq. (17):

$$
\begin{aligned}
\overline{\left\langle\psi\left|\rho_{\text {out }}\right| \psi\right\rangle} & =p_{I}+\frac{p_{X}+p_{Y}+p_{Z}}{2}+O\left(\left(m e^{-2 \eta|\alpha|^{2}}\right)^{n}\right) \\
& =p_{I}+\frac{p_{Z}}{2}+O\left(\left(m e^{-2 \eta|\alpha|^{2}}\right)^{n}\right),
\end{aligned}
$$

where

$$
\begin{aligned}
4 p_{I}:= & \sum_{i}\left\langle B_{i}\left|M_{i}\right| B_{i}\right\rangle, \\
4 p_{X}:= & \left\langle B_{2}\left|M_{0}\right| B_{2}\right\rangle+\left\langle B_{3}\left|M_{1}\right| B_{3}\right\rangle \\
& +\left\langle B_{0}\left|M_{2}\right| B_{0}\right\rangle+\left\langle B_{1}\left|M_{3}\right| B_{1}\right\rangle, \\
4 p_{Y}:= & \left\langle B_{3}\left|M_{0}\right| B_{3}\right\rangle+\left\langle B_{2}\left|M_{1}\right| B_{2}\right\rangle \\
& +\left\langle B_{1}\left|M_{2}\right| B_{1}\right\rangle+\left\langle B_{0}\left|M_{3}\right| B_{0}\right\rangle, \\
4 p_{Z}:= & \left\langle B_{1}\left|M_{0}\right| B_{1}\right\rangle+\left\langle B_{0}\left|M_{1}\right| B_{0}\right\rangle \\
& +\left\langle B_{3}\left|M_{2}\right| B_{3}\right\rangle+\left\langle B_{2}\left|M_{3}\right| B_{2}\right\rangle .
\end{aligned}
$$

The second equality in Eq. (D5) holds due to $\eta_{1}=\eta_{2}$.

\section{APPENDIX E: CLOSED-FORM EXPRESSION OF $N_{\text {exp,SCS }}$ AND $N_{\text {exp,g }}$}

Here we get closed-form expressions of $N_{\text {exp,SCs }}$ and $N_{\text {exp,g }}$ defined in Sec. VII A 2, the expected numbers of trials of creating an SCS or implementing a CNOT gate to encode a logical qubit, in terms of the success probabilities $\left(p_{\mathrm{s}, \mathrm{SCS}}\right.$ and $\left.p_{\mathrm{s}, \mathrm{g}}\right)$ of the schemes to generate an SCS and to implement a CNOT gate. We first assume $p_{\mathrm{s}, \mathrm{SCS}}=1$ and denote $p:=p_{\mathrm{s}, \mathrm{g}}$ for simplicity. From the encoding circuit in Fig. 11, it is observed that the first $i$ CNOT gates are involved with $\min (i, n)$ blocks and each block uses $m-1$ SCSs. Hence, if the $i$ th CNOT gate fails, one should prepare new $(m-1) \min (i, n)$ SCSs and try the encoding again.

For each trial of encoding, there are $n m$ possible events, each of which is denoted as $E_{\mathrm{s}}$ or $E_{i}$ for $1 \leqslant i \leqslant n m-1$. The event $E_{\mathrm{s}}\left(E_{i}\right)$ corresponds to the success (failure) of the $i$ th CNOT gate. The probability of each $E_{i}$ and the corresponding numbers ( $N_{\mathrm{SCS}}$ and $N_{\mathrm{g}}$ ) of trials of creating an SCS and implementing a CNOT gate are given as follows:

$$
\begin{aligned}
& \operatorname{Pr}\left(E_{\mathrm{s}}\right)=p^{n m-1}, \\
& \operatorname{Pr}\left(E_{i}\right)=p^{i-1}(1-p),
\end{aligned}
$$

$$
\begin{aligned}
N_{\mathrm{SCS}}\left(E_{\mathrm{S}}\right) & =n(m-1), \\
N_{\mathrm{SCS}}\left(E_{i}\right) & =(m-1) \min (i, n), \\
N_{\mathrm{g}}\left(E_{\mathrm{S}}\right) & =n m-1, \\
N_{\mathrm{g}}\left(E_{i}\right) & =i .
\end{aligned}
$$

From these results, we get the expectation of $N_{\mathrm{SCS}}$ conditioning that we do not get $E_{\mathrm{s}}$ :

$$
\begin{aligned}
\left\langle N_{\mathrm{SCS}}\right\rangle_{\text {fail }} & =\frac{\sum_{i} N_{\mathrm{SCS}}\left(E_{i}\right) \operatorname{Pr}\left(E_{i}\right)}{1-\operatorname{Pr}\left(E_{s}\right)} \\
& =(m-1)\left[n-\frac{1}{1-p^{n m-1}}\left(n-\frac{1-p^{n}}{1-p}\right)\right] .
\end{aligned}
$$

Similarly, we get

$$
\left\langle N_{\mathrm{g}}\right\rangle_{\mathrm{fail}}=\frac{1}{1-p}-\frac{(n m-1) p^{n m-1}}{1-p^{n m-1}} .
$$

We now consider repeating multiple trials of encoding until we get $E_{\mathrm{s}}$. The expected number of failed events before the successful one is $1 / \operatorname{Pr}\left(E_{\mathrm{s}}\right)-1=1 / p^{n m-1}-1$. We thus obtain $N_{\text {exp,SCS }}$ and $N_{\text {exp,g }}$ as follows:

$$
\begin{aligned}
N_{\text {exp }, \text { SCS }} & =\left(\frac{1}{p^{n m-1}}-1\right)\left\langle N_{\mathrm{SCS}}\right\rangle_{\text {fail }}+n(m-1) \\
& =(m-1)\left[\frac{1-p^{n}}{p^{n m-1}(1-p)}\right], \\
N_{\text {exp }, \mathrm{g}} & =\left(\frac{1}{p^{n m-1}}-1\right)\left\langle N_{\mathrm{g}}\right\rangle_{\text {fail }}+n m-1 \\
& =\frac{1-p^{n m-1}}{p^{n m-1}(1-p)} .
\end{aligned}
$$

If $p_{\mathrm{s}, \mathrm{SCS}} \leqslant 1,1 / p_{\mathrm{s}, \mathrm{SCS}}$ trials are on average required to create a SCS. Therefore, we can rewrite $N_{\text {exp,SCS }}$ as

$$
N_{\text {exp }, \mathrm{SCS}}=\frac{m-1}{p_{\mathrm{s}, \mathrm{SCS}}}\left[\frac{1-p^{n}}{p^{n m-1}(1-p)}\right] .
$$

Equations (E1) and (E2) are the closed-form expressions that we want.

\section{APPENDIX F: CLOSED-FORM EXPRESSION OF $F_{\eta}^{\text {avg }}$}

Here we get a closed-form expression of $F_{\eta}^{\text {avg }}$ defined in Sec. VII A 2 as

$$
\begin{aligned}
F_{\eta}^{\text {avg }}: & =\overline{F\left(\Lambda_{\eta}(|\psi\rangle\langle\psi|),|\psi\rangle\langle\psi|\right)} \\
& =\overline{\left\langle\psi\left|\Lambda_{\eta}(|\psi\rangle\langle\psi|)\right| \psi\right\rangle}
\end{aligned}
$$

(omitting the subscripts $L$ ), where

$$
\overline{f(|\psi\rangle)}:=\frac{1}{4 \pi} \int_{0}^{\pi} d \theta \sin \theta \int_{0}^{2 \pi} d \phi f(|\psi\rangle)
$$

for a function $f$ and

$$
|\psi\rangle:=\frac{1}{\sqrt{1+R \sin \theta \cos \phi}}\left(\cos \frac{\theta}{2}|0\rangle+e^{i \phi} \sin \frac{\theta}{2}|1\rangle\right)
$$

with $R:=\langle 0 \mid 1\rangle$. The effect of the photon loss channel $\Lambda_{\eta}$ is shown in Eq. (A1). 
$F_{\eta}^{\text {avg }}$ can be expressed as

$$
F_{\eta}^{\mathrm{avg}}=\sum_{i, j, k, l} c_{i j k l}\left\langle i\left|\Lambda_{\eta}(|j\rangle\langle j| k)\right| l\right\rangle .
$$

Calculating the integrals, we get

$$
\begin{aligned}
c_{0000} & =c_{1111} \\
& =\frac{1}{4 R^{2}}\left[\frac{1}{1-R^{2}}+\frac{1}{2 R} \ln \left(\frac{1+R}{1-R}\right)-2\right]:=\frac{f_{1}}{4 R^{2}}, \\
c_{1001} & =c_{0110}=c_{0011} \\
& =\frac{1}{4 R^{2}}\left[\frac{1}{1-R^{2}}-\frac{1}{2 R} \ln \left(\frac{1+R}{1-R}\right)\right]:=\frac{f_{2}}{4 R^{2}}, \\
c_{0001} & =c_{0111}=c_{0010}=c_{1011}=-\frac{f_{2}}{4 R}, \\
c_{1010} & =\frac{1}{4 R^{2}}\left[2+\frac{1}{1-R^{2}}-\frac{3}{2 R} \ln \left(\frac{1+R}{1-R}\right)\right]:=\frac{f_{3}}{4 R^{2}},
\end{aligned}
$$

and $\forall i, j, k, l, \quad c_{i j k l}=c_{l k j i}$. Next, $\left\langle i\left|\Lambda_{\eta}(|j\rangle\langle j| k)\right| l\right\rangle$ is calculated as

$$
\begin{aligned}
&\left\langle 0\left|\Lambda_{\eta}(|0\rangle\langle 0| 0)\right| 0\right\rangle=\left\langle 1\left|\Lambda_{\eta}(|1\rangle\langle 1| 1)\right| 1\right\rangle=C a_{+} b_{+}, \\
&\left\langle 1\left|\Lambda_{\eta}(|0\rangle\langle 0| 0)\right| 1\right\rangle=\left\langle 0\left|\Lambda_{\eta}(|1\rangle\langle 1| 1)\right| 0\right\rangle=C a_{+} b_{-}, \\
&\left\langle 0\left|\Lambda_{\eta}(|0\rangle\langle 0| 1)\right| 1\right\rangle=C a_{-} b_{+}, \\
&\left\langle 0\left|\Lambda_{\eta}(|0\rangle\langle 0| 0)\right| 1\right\rangle=\left\langle 1\left|\Lambda_{\eta}(|1\rangle\langle 1| 1)\right| 0\right\rangle=C a_{+} d,
\end{aligned}
$$

$$
\begin{aligned}
\left\langle 0\left|\Lambda_{\eta}(|0\rangle\langle 0| 1)\right| 0\right\rangle= & \left\langle 1\left|\Lambda_{\eta}(|0\rangle\langle 0| 1)\right| 1\right\rangle=C a_{-} d, \\
& \left\langle 1\left|\Lambda_{\eta}(|0\rangle\langle 0| 1)\right| 0\right\rangle=C a_{-} b_{-},
\end{aligned}
$$

where

$$
\begin{aligned}
x:= & e^{-|\alpha|^{2}}, \\
C:= & {\left[\frac{2}{\left(1+x^{2}\right)^{m}+\left(1-x^{2}\right)^{m}}\right]^{n}, } \\
a_{ \pm}:= & {\left[\left(\frac{1+x^{2\left(1-\eta^{2}\right)}}{2}\right)^{m} \pm\left(\frac{1-x^{2\left(1-\eta^{2}\right)}}{2}\right)^{m}\right]^{n}, } \\
b_{ \pm}:= & \frac{1}{4^{n}}\left[\left(x^{(1-\eta)^{2}}+x^{(1+\eta)^{2}}+2 x^{\left(1+\eta^{2}\right)}\right)^{m}\right. \\
& +\left(x^{(1-\eta)^{2}}+x^{(1+\eta)^{2}}-2 x^{\left(1+\eta^{2}\right)}\right)^{m} \\
& \left. \pm 2\left(x^{(1-\eta)^{2}}-x^{(1+\eta)^{2}}\right)^{m}\right]^{n}, \\
d:= & \frac{1}{4^{n}}\left[\left(x^{(1-\eta)^{2}}+x^{(1+\eta)^{2}}+2 x^{\left(1+\eta^{2}\right)}\right)^{m}\right. \\
& \left.-\left(x^{(1-\eta)^{2}}+x^{(1+\eta)^{2}}-2 x^{\left(1+\eta^{2}\right)}\right)^{m}\right]^{n} .
\end{aligned}
$$

Combining the above results, we obtain the following closedform expression of $F_{\eta}^{\text {avg }}$ in terms of $\alpha, R, \eta, n$, and $m$ :

$$
\begin{aligned}
F_{\eta}^{\mathrm{avg}}= & \frac{C}{2 R^{2}}\left[f_{1} a_{+} b_{+}+f_{3} a_{-} b_{-}\right. \\
& \left.+f_{2}\left\{a_{+} b_{-}+a_{-} b_{+}-2 R\left(a_{+}+a_{-}\right) d\right\}\right] .
\end{aligned}
$$

[1] P. Kok and B. W. Lovett, Introduction to Optical Quantum Information Processing (Cambridge University Press, Cambridge, 2010).

[2] A. P. Lund and T. C. Ralph, Nondeterministic gates for photonic single-rail quantum logic, Phys. Rev. A 66, 032307 (2002).

[3] E. Knill, R. Laflamme, and G. J. Milburn, A scheme for efficient quantum computation with linear optics, Nature (London) 409, 46 (2001).

[4] N. Lütkenhaus, J. Calsamiglia, and K.-A. Suominen, Bell measurements for teleportation, Phys. Rev. A 59, 3295 (1999).

[5] J. Calsamiglia and N. Lütkenhaus, Maximum efficiency of a linear-optical Bell-state analyzer, Appl. Phys. B 72, 67 (2001).

[6] C. H. Bennett, G. Brassard, C. Crépeau, R. Jozsa, A. Peres, and W. K. Wootters, Teleporting an Unknown Quantum State Via Dual Classical and Einstein-Podolsky-Rosen Channels, Phys. Rev. Lett. 70, 1895 (1993).

[7] D. Gottesman and I. L. Chuang, Demonstrating the viability of universal quantum computation using teleportation and singlequbit operations, Nature (London) 402, 390 (1999).

[8] M. Zukowski, A. Zeilinger, M. A. Horne, and A. K. Ekert, "Event-Ready-Detectors" Bell Experiment Via Entanglement Swapping, Phys. Rev. Lett. 71, 4287 (1993).

[9] J.-W. Pan, D. Bouwmeester, H. Weinfurter, and A. Zeilinger, Experimental Entanglement Swapping: Entangling Photons that Never Interacted, Phys. Rev. Lett. 80, 3891 (1998).
[10] S.-W. Lee, K. Park, T. C. Ralph, and H. Jeong, Nearly Deterministic Bell Measurement for Multiphoton Qubits and Its Application to Quantum Information Processing, Phys. Rev. Lett. 114, 113603 (2015).

[11] S.-W. Lee, T. C. Ralph, and H. Jeong, Fundamental building block for all-optical scalable quantum networks, Phys. Rev. A 100, 052303 (2019).

[12] W. P. Grice, Arbitrarily complete Bell-state measurement using only linear optical elements, Phys. Rev. A 84, 042331 (2011).

[13] H. A. Zaidi and P. van Loock, Beating the One-Half Limit of Ancilla-Free Linear Optics Bell Measurements, Phys. Rev. Lett. 110, 260501 (2013).

[14] F. Ewert and P. van Loock, 3/4-efficient Bell Measurement with Passive Linear Optics and Unentangled Ancillae, Phys. Rev. Lett. 113, 140403 (2014).

[15] H. Jeong, M. S. Kim, and J. Lee, Quantum-information processing for a coherent superposition state via a mixed entangled coherent channel, Phys. Rev. A 64, 052308 (2001).

[16] H. Jeong and M. S. Kim, Purification of entangled coherent states, Quantum Inf. Comput. 2, 208 (2002), arXiv:quant$\mathrm{ph} / 0111015$.

[17] H. Jeong and M. S. Kim, Efficient quantum computation using coherent states, Phys. Rev. A 65, 042305 (2002).

[18] T. C. Ralph, A. Gilchrist, G. J. Milburn, W. J. Munro, and S. Glancy, Quantum computation with optical coherent states, Phys. Rev. A 68, 042319 (2003). 
[19] S. Glancy, H. M. Vasconcelos, and T. C. Ralph, Transmission of optical coherent-state qubits, Phys. Rev. A 70, 022317 (2004).

[20] S. L. Braunstein and P. van Loock, Quantum information with continuous variables, Rev. Mod. Phys. 77, 513 (2005).

[21] H. Jeong and T. C. Ralph, Schrödinger cat states for quantum information processing, in Quantum Information with Continuous Variables of Atoms and Light (World Scientific, Singapore, 2007), pp. 159-179.

[22] A. P. Lund, T. C. Ralph, and H. L. Haselgrove, Fault-Tolerant Linear Optical Quantum Computing with Small-Amplitude Coherent States, Phys. Rev. Lett. 100, 030503 (2008).

[23] J. S. Neergaard-Nielsen, Y. Eto, C.-W. Lee, H. Jeong, and M. Sasaki, Quantum tele-amplification with a continuous-variable superposition state, Nat. Photon. 7, 439 (2013).

[24] M. Mirrahimi, Z. Leghtas, V. V. Albert, S. Touzard, R. J. Schoelkopf, L. Jiang, and M. H. Devoret, Dynamically protected cat-qubits: a new paradigm for universal quantum computation, New J. Phys. 16, 045014 (2014).

[25] S.-W. Lee and H. Jeong, Near-deterministic quantum teleportation and resource-efficient quantum computation using linear optics and hybrid qubits, Phys. Rev. A 87, 022326 (2013).

[26] S. Choi, S.-H. Lee, and H. Jeong, Teleportation of a multiphoton qubit using hybrid entanglement with a loss-tolerant carrier qubit, Phys. Rev. A 102, 012424 (2020).

[27] S. Omkar, Y. S. Teo, and H. Jeong, Resource-Efficient Topological Fault-Tolerant Quantum Computation with Hybrid Entanglement of Light, Phys. Rev. Lett. 125, 060501 (2020).

[28] S.-W. Lee and H. Jeong, Bell-state measurement and quantum teleportation using linear optics: two-photon pairs, entangled coherent states, and hybrid entanglement, in Proceedings of the First International Workshop on Entangled Coherent States and Its Application to Quantum Information Science; Towards Macroscopic Quantum Communications (Tokyo, Japan, 2013), pp. $41-46$.

[29] P. T. Cochrane, G. J. Milburn, and W. J. Munro, Macroscopically distinct quantum-superposition states as a bosonic code for amplitude damping, Phys. Rev. A 59, 2631 (1999).

[30] S. Lloyd and S. L. Braunstein, Quantum computation over continuous variables, in Quantum Information with Continuous Variables (Springer, Dordrecht, 1999), pp. 9-17.

[31] M. C. de Oliveira and W. J. Munro, Quantum computation with mesoscopic superposition states, Phys. Rev. A 61, 042309 (2000).

[32] S. D. Bartlett, H. de Guise, and B. C. Sanders, Quantum encodings in spin systems and harmonic oscillators, Phys. Rev. A 65, 052316 (2002).

[33] Z. Leghtas, G. Kirchmair, B. Vlastakis, R. J. Schoelkopf, M. H. Devoret, and M. Mirrahimi, Hardware-Efficient Autonomous Quantum Memory Protection, Phys. Rev. Lett. 111, 120501 (2013).

[34] V. V. Albert, C. Shu, S. Krastanov, C. Shen, R.-B. Liu, Z.-B. Yang, R. J. Schoelkopf, M. Mirrahimi, M. H. Devoret, and L. Jiang, Holonomic Quantum Control with Continuous Variable Systems, Phys. Rev. Lett. 116, 140502 (2016).

[35] S. Puri, S. Boutin, and A. Blais, Engineering the quantum states of light in a kerr-nonlinear resonator by two-photon driving, npj Quantum Inf. 3, 18 (2017).

[36] L. Li, C.-L. Zou, V. V. Albert, S. Muralidharan, S. M. Girvin, and L. Jiang, Cat Codes with Optimal Decoherence Sup- pression for a Lossy Bosonic Channel, Phys. Rev. Lett. 119, 030502 (2017).

[37] J. Cohen, W. C. Smith, M. H. Devoret, and M. Mirrahimi, Degeneracy-Preserving Quantum Nondemolition Measurement of Parity-Type Observables for Cat Qubits, Phys. Rev. Lett. 119, 060503 (2017).

[38] S. Puri, A. Grimm, P. Campagne-Ibarcq, A. Eickbusch, K. Noh, G. Roberts, L. Jiang, M. Mirrahimi, M. H. Devoret, and S. M. Girvin, Stabilized Cat in a Driven Nonlinear Cavity: A Fault-Tolerant Error Syndrome Detector, Phys. Rev. X 9, 041009 (2019).

[39] Z. Leghtas, S. Touzard, I. M. Pop, A. Kou, B. Vlastakis, A. Petrenko, K. M. Sliwa, A. Narla, S. Shankar, M. J. Hatridge, M. Reagor, L. Frunzio, R. J. Schoelkopf, M. Mirrahimi, and M. H. Devoret, Confining the state of light to a quantum manifold by engineered two-photon loss, Science 347, 853 (2015).

[40] N. Ofek, A. Petrenko, R. Heeres, P. Reinhold, Z. Leghtas, B. Vlastakis, Y. Liu, L. Frunzio, S. M. Girvin, L. Jiang, M. Mirrahimi, M. H. Devoret, and R. J. Schoelkopf, Extending the lifetime of a quantum bit with error correction in superconducting circuits, Nature (London) 536, 441 (2016).

[41] S. Touzard, A. Grimm, Z. Leghtas, S. O. Mundhada, P. Reinhold, C. Axline, M. Reagor, K. Chou, J. Blumoff, K. M. Sliwa, S. Shankar, L. Frunzio, R. J. Schoelkopf, M. Mirrahimi, and M. H. Devoret, Coherent Oscillations Inside a Quantum Manifold Stabilized by Dissipation, Phys. Rev. X 8, 021005 (2018).

[42] S. Rosenblum, P. Reinhold, M. Mirrahimi, L. Jiang, L. Frunzio, and R. J. Schoelkopf, Fault-tolerant detection of a quantum error, Science 361, 266 (2018).

[43] J. Guillaud and M. Mirrahimi, Repetition Cat Qubits for Fault-Tolerant Quantum Computation, Phys. Rev. X 9, 041053 (2019).

[44] S. Muralidharan, J. Kim, N. Lütkenhaus, M. D. Lukin, and L. Jiang, Ultrafast and Fault-Tolerant Quantum Communication Across Long Distances, Phys. Rev. Lett. 112, 250501 (2014)

[45] N. Sangouard, C. Simon, H. de Riedmatten, and N. Gisin, Quantum repeaters based on atomic ensembles and linear optics, Rev. Mod. Phys. 83, 33 (2011).

[46] H.-J. Briegel, W. Dür, J. I. Cirac, and P. Zoller, Quantum Repeaters: The Role of Imperfect Local Operations in Quantum Communication, Phys. Rev. Lett. 81, 5932 (1998).

[47] W. Dür, H.-J. Briegel, J. I. Cirac, and P. Zoller, Quantum repeaters based on entanglement purification, Phys. Rev. A 59, 169 (1999).

[48] L.-M. Duan, M. D. Lukin, J. I. Cirac, and P. Zoller, Longdistance quantum communication with atomic ensembles and linear optics, Nature (London) 414, 413 (2001).

[49] P. Kok, C. P. Williams, and J. P. Dowling, Construction of a quantum repeater with linear optics, Phys. Rev. A 68, 022301 (2003).

[50] C. Simon, H. de Riedmatten, M. Afzelius, N. Sangouard, H. Zbinden, and N. Gisin, Quantum Repeaters with Photon Pair Sources and Multimode Memories, Phys. Rev. Lett. 98, 190503 (2007).

[51] S. Muralidharan, L. Li, J. Kim, N. Lütkenhaus, M. D. Lukin, and L. Jiang, Optimal architectures for long distance quantum communication, Sci. Rep. 6, 20463 (2016). 
[52] L. Jiang, J. M. Taylor, K. Nemoto, W. J. Munro, R. Van Meter, and M. D. Lukin, Quantum repeater with encoding, Phys. Rev. A 79, 032325 (2009).

[53] W. J. Munro, K. A. Harrison, A. M. Stephens, S. J. Devitt, and K. Nemoto, From quantum multiplexing to high-performance quantum networking, Nat. Photon. 4, 792 (2010).

[54] N. Sangouard, C. Simon, N. Gisin, J. Laurat, R. Tualle-Brouri, and P. Grangier, Quantum repeaters with entangled coherent states, J. Opt. Soc. Am. B 27, A137 (2010).

[55] W. J. Munro, A. M. Stephens, S. J. Devitt, K. A. Harrison, and K. Nemoto, Quantum communication without the necessity of quantum memories, Nat. Photon. 6, 777 (2012).

[56] K. Azuma, K. Tamaki, and H.-K. Lo, All-photonic quantum repeaters, Nat. Commun. 6, 6787 (2015).

[57] M. Zwerger, H. J. Briegel, and W. Dür, Measurement-based quantum communication, Appl. Phys. B 122, 50 (2016).

[58] F. Ewert, M. Bergmann, and P. van Loock, Ultrafast LongDistance Quantum Communication with Static Linear Optics, Phys. Rev. Lett. 117, 210501 (2016).

[59] S. J. D. Phoenix, Wave-packet evolution in the damped oscillator, Phys. Rev. A 41, 5132 (1990).

[60] U. Leonhardt, Quantum statistics of a lossless beam splitter: SU(2) symmetry in phase space, Phys. Rev. A 48, 3265 (1993).

[61] T. C. Ralph, A. J. F. Hayes, and A. Gilchrist, Loss-Tolerant Optical Qubits, Phys. Rev. Lett. 95, 100501 (2005).

[62] V. Scarani, H. Bechmann-Pasquinucci, N. J. Cerf, M. Dušek, N. Lütkenhaus, and M. Peev, The security of practical quantum key distribution, Rev. Mod. Phys. 81, 1301 (2009).

[63] For CBSM with multi-photon polarization qubits, we use $C_{\exp }=n m$, the number of physical-level BSMs for one CBSM, in the definition of $Q_{\text {tot }}$ [see Eq. (19)].

[64] D. Bures, An extension of Kakutani's theorem on infinite product measures to the tensor product of semifinite $\mathrm{w}^{*}$-algebras, Trans. Amer. Math. Soc. 135, 199 (1969).

[65] B. Yurke and D. Stoler, Generating Quantum Mechanical Superpositions of Macroscopically Distinguishable States Via Amplitude Dispersion, Phys. Rev. Lett. 57, 13 (1986).

[66] M. Dakna, T. Anhut, T. Opatrný, L. Knöll, and D.-G. Welsch, Generating Schrödinger-cat-like states by means of conditional measurements on a beam splitter, Phys. Rev. A 55, 3184 (1997).

[67] S. Song, C. M. Caves, and B. Yurke, Generation of superpositions of classically distinguishable quantum states from optical back-action evasion, Phys. Rev. A 41, 5261 (1990).

[68] A. P. Lund, H. Jeong, T. C. Ralph, and M. S. Kim, Conditional production of superpositions of coherent states with inefficient photon detection, Phys. Rev. A 70, 020101(R) (2004).

[69] H. Jeong, A. P. Lund, and T. C. Ralph, Production of superpositions of coherent states in traveling optical fields with inefficient photon detection, Phys. Rev. A 72, 013801 (2005).

[70] H. Jeong, M. S. Kim, T. C. Ralph, and B. S. Ham, Generation of macroscopic superposition states with small nonlinearity, Phys. Rev. A 70, 061801(R) (2004).

[71] S. D. Barrett, P. Kok, K. Nemoto, R. G. Beausoleil, W. J. Munro, and T. P. Spiller, Symmetry analyzer for nondestructive Bell-state detection using weak nonlinearities, Phys. Rev. A 71, 060302(R) (2005).

[72] H. Jeong, Using weak nonlinearity under decoherence for macroscopic entanglement generation and quantum computation, Phys. Rev. A 72, 034305 (2005).
[73] A. Ourjoumtsev, H. Jeong, R. Tualle-Brouri, and P. Grangier, Generation of optical 'Schrödinger cats' from photon number states, Nature (London) 448, 784 (2007).

[74] A. Ourjoumtsev, R. Tualle-Brouri, J. Laurat, and P. Grangier, Generating optical Schrödinger kittens for quantum information processing, Science 312, 83 (2006).

[75] T. Gerrits, S. Glancy, T. S. Clement, B. Calkins, A. E. Lita, A. J. Miller, A. L. Migdall, S. W. Nam, R. P. Mirin, and E. Knill, Generation of optical coherent-state superpositions by number-resolved photon subtraction from the squeezed vacuum, Phys. Rev. A 82, 031802(R) (2010).

[76] J. S. Neergaard-Nielsen, M. Takeuchi, K. Wakui, H. Takahashi, K. Hayasaka, M. Takeoka, and M. Sasaki, Optical Continuous-Variable Qubit, Phys. Rev. Lett. 105, 053602 (2010).

[77] W. Asavanant, K. Nakashima, Y. Shiozawa, J.-I. Yoshikawa, and A. Furusawa, Generation of highly pure Schrödinger's cat states and real-time quadrature measurements via optical filtering, Opt. Express 25, 32227 (2017).

[78] D. V. Sychev, A. E. Ulanov, A. A. Pushkina, M. W. Richards, I. A. Fedorov, and A. I. Lvovsky, Enlargement of optical Schrödinger's cat states, Nat. Photon. 11, 379 (2017).

[79] T. Serikawa, J.-i. Yoshikawa, S. Takeda, H. Yonezawa, T. C. Ralph, E. H. Huntington, and A. Furusawa, Generation of a Cat State in an Optical Sideband, Phys. Rev. Lett. 121, 143602 (2018).

[80] A. Laghaout, J. S. Neergaard-Nielsen, I. Rigas, C. Kragh, A. Tipsmark, and U. L. Andersen, Amplification of realistic Schrödinger-cat-state-like states by homodyne heralding, Phys. Rev. A 87, 043826 (2013).

[81] P. Marek and J. Fiurášek, Elementary gates for quantum information with superposed coherent states, Phys. Rev. A 82, 014304 (2010).

[82] A. Tipsmark, R. Dong, A. Laghaout, P. Marek, M. Ježek, and U. L. Andersen, Experimental demonstration of a Hadamard gate for coherent state qubits, Phys. Rev. A 84, 050301(R) (2011).

[83] S. Haroche, M. Brune, and J.-M. Raimond, Measuring the photon number parity in a cavity: from light quantum jumps to the tomography of non-classical field states, J. Mod. Opt. 54, 2101 (2007).

[84] L. Sun, A. Petrenko, Z. Leghtas, B. Vlastakis, G. Kirchmair, K. M. Sliwa, A. Narla, M. Hatridge, S. Shankar, J. Blumoff, L. Frunzio, M. Mirrahimi, M. H. Devoret, and R. J. Schoelkopf, Tracking photon jumps with repeated quantum nondemolition parity measurements, Nature (London) 511, 444 (2014).

[85] C. C. Gerry, A. Benmoussa, and R. A. Campos, Quantum nondemolition measurement of parity and generation of parity eigenstates in optical fields, Phys. Rev. A 72, 053818 (2005).

[86] C. C. Gerry and J. Mimih, The parity operator in quantum optical metrology, Contemp. Phys. 51, 497 (2010).

[87] J.-C. Besse, S. Gasparinetti, M. C. Collodo, T. Walter, A. Remm, J. Krause, C. Eichler, and A. Wallraff, Parity Detection of Propagating Microwave Fields, Phys. Rev. X 10, 011046 (2020).

[88] M. Jönsson and G. Björk, Evaluating the performance of photon-number-resolving detectors, Phys. Rev. A 99, 043822 (2019). 
[89] B. Cabrera, R. M. Clarke, P. Colling, A. J. Miller, S. Nam, and R. W. Romani, Detection of single infrared, optical, and ultraviolet photons using superconducting transition edge sensors, Appl. Phys. Lett. 73, 735 (1998).

[90] A. J. Miller, S. W. Nam, J. M. Martinis, and A. V. Sergienko, Demonstration of a low-noise near-infrared photon counter with multiphoton discrimination, Appl. Phys. Lett. 83, 791 (2003).

[91] A. E. Lita, A. J. Miller, and S. W. Nam, Counting near-infrared single-photons with 95\% efficiency, Opt. Express 16, 3032 (2008).

[92] F. Marsili, V. B. Verma, J. A. Stern, S. Harrington, A. E. Lita, T. Gerrits, I. Vayshenker, B. Baek, M. D. Shaw, R. P. Mirin, and S. W. Nam, Detecting single infrared photons with $93 \%$ system efficiency, Nat. Photon. 7, 210 (2013).

[93] G. Harder, T. J. Bartley, A. E. Lita, S. W. Nam, T. Gerrits, and C. Silberhorn, Single-Mode Parametric-Down-Conversion States with 50 Photons as a Source for Mesoscopic Quantum Optics, Phys. Rev. Lett. 116, 143601 (2016).

[94] J. Sperling, W. R. Clements, A. Eckstein, M. Moore, J. J. Renema, W. S. Kolthammer, S. W. Nam, A. Lita, T. Gerrits, W. Vogel, G. S. Agarwal, and I. A. Walmsley, DetectorIndependent Verification of Quantum Light, Phys. Rev. Lett. 118, 163602 (2017).

[95] G. Lubin, R. Tenne, I. M. Antolovic, E. Charbon, C. Bruschini, and D. Oron, Quantum correlation measurement with single photon avalanche diode arrays, Opt. Express 27, 32863 (2019).
[96] M. J. Fitch, B. C. Jacobs, T. B. Pittman, and J. D. Franson, Photon-number resolution using time-multiplexed singlephoton detectors, Phys. Rev. A 68, 043814 (2003).

[97] D. Achilles, C. Silberhorn, C. Śliwa, K. Banaszek, and I. A. Walmsley, Fiber-assisted detection with photon number resolution, Opt. Lett. 28, 2387 (2003).

[98] D. Achilles, C. Silberhorn, C. Sliwa, K. Banaszek, I. A. Walmsley, M. J. Fitch, B. C. Jacobs, T. B. Pittman, and J. D. Franson, Photon-number-resolving detection using timemultiplexing, J. Mod. Opt. 51, 1499 (2004).

[99] A. Divochiy, F. Marsili, D. Bitauld, A. Gaggero, R. Leoni, F. Mattioli, A. Korneev, V. Seleznev, N. Kaurova, O. Minaeva, G. Gol'tsman, K. G. Lagoudakis, M. Benkhaoul, F. Lévy, and A. Fiore, Superconducting nanowire photon-number-resolving detector at telecommunication wavelengths, Nat. Photon. 2, 302 (2008).

[100] F. Mattioli, Z. Zhou, A. Gaggero, R. Gaudio, S. Jahanmirinejad, D. Sahin, F. Marsili, R. Leoni, and A. Fiore, Photon-number-resolving superconducting nanowire detectors, Supercond. Sci. Technol. 28, 104001 (2015).

[101] R. Nehra, C.-H. Chang, Q. Yu, A. Beling, and O. Pfister, Photon-number-resolving segmented detectors based on single-photon avalanche-photodiodes, Opt. Express 28, 3660 (2020).

[102] R. Blandino, F. Ferreyrol, M. Barbieri, P. Grangier, and R. Tualle-Brouri, Characterization of a $\pi$-phase shift quantum gate for coherent-state qubits, New J. Phys. 14, 013017 (2012). 